\title{
On the use of ion beams to emulate the neutron irradiation behaviour of tungsten
}

DOI:

10.1016/j.vacuum.2018.11.050

\section{Document Version}

Accepted author manuscript

Link to publication record in Manchester Research Explorer

\section{Citation for published version (APA):}

Harrison, R. W. (2019). On the use of ion beams to emulate the neutron irradiation behaviour of tungsten.

Vacuum, 160, 355-370. https://doi.org/10.1016/j.vacuum.2018.11.050

\section{Published in:}

Vacuum

\section{Citing this paper}

Please note that where the full-text provided on Manchester Research Explorer is the Author Accepted Manuscript or Proof version this may differ from the final Published version. If citing, it is advised that you check and use the publisher's definitive version.

\section{General rights}

Copyright and moral rights for the publications made accessible in the Research Explorer are retained by the authors and/or other copyright owners and it is a condition of accessing publications that users recognise and abide by the legal requirements associated with these rights.

\section{Takedown policy}

If you believe that this document breaches copyright please refer to the University of Manchester's Takedown Procedures [http://man.ac.uk/04Y6Bo] or contact uml.scholarlycommunications@manchester.ac.uk providing relevant details, so we can investigate your claim.

\section{OPEN ACCESS}




\title{
On the use of ion beams to emulate the neutron irradiation behaviour of tungsten
}

\author{
R.W. Harrison ${ }^{1}$ \\ School of Computing and Engineering, University of Huddersfield, Queensgate, \\ Huddersfield, HD1 3DH, UK
}

\begin{abstract}
Tungsten (W) is the primary candidate for use as the divertor armour in magnetic confinement nuclear fusion reactors. As such, the material will be plasma facing and subject to extremely harsh environments due to neutron and charged particle bombardment. To predict the in-service behaviour of tungsten during operation, ion beams are extensively used as a proxy for neutron irradiation damage. However, reproducing the complexities of radiation damage mechanisms as well as transmutation effects of neutron irradiations of $\mathrm{W}$ with single or multiple ion beams is still in its infancy and results can often seem contradictory. This review paper aims to collate and unite these disparate observations, with the aim of aiding the communities when moving forward to use the state-of-the-art in ion beam technology to emulate neutron damage in tungsten.
\end{abstract}

Keywords: Tungsten, Irradiation, Fusion, Neutron, Ion beam,

\section{Introduction}

At the temperatures required for fusion matter becomes ionised plasma, which may be contained within a toroidal magnetic field. Nuclear fusion reactions (D-T) produce high energy $14.1 \mathrm{MeV}$ neutrons and 3.5 MeV $\alpha$-particles [1]. Whilst the energy of $\alpha$-particles lost from the plasma as a result of D-T reactions will generally be around $10 \mathrm{~s}$ of $\mathrm{eV}$, particles with $\mathrm{MeV}$ energies have been measured escaping the plasma confinement of the Joint European Torus (JET) reactor [2]. Tungsten (W) has been used successfully as a plasma-facing material in fusion experiments in the Joint European Torus (JET) reactor (see Figure 1a); is regarded as the primary candidate for use as a as

\footnotetext{
${ }^{1}$ Current email and address; R.W.Harrison@Manchester.ac.uk, School of Mechanical, Aerospace and Civil Engineering, The University of Manchester, Sackville Street, Manchester, M1 3NJ, UK
} 
the divertor armour (see Figure 1b) in ITER and foreseeably its successor, the DEMOnstration (DEMO) fusion reactors. Its widespread use is due to its high melting temperature $(\sim 3700 \mathrm{~K})$, low sputter yield and high thermal conductivity $\left(\sim 170 \mathrm{~W} \cdot \mathrm{m}^{-1} \cdot \mathrm{K}^{-1}\right.$ at room temperature) [1].

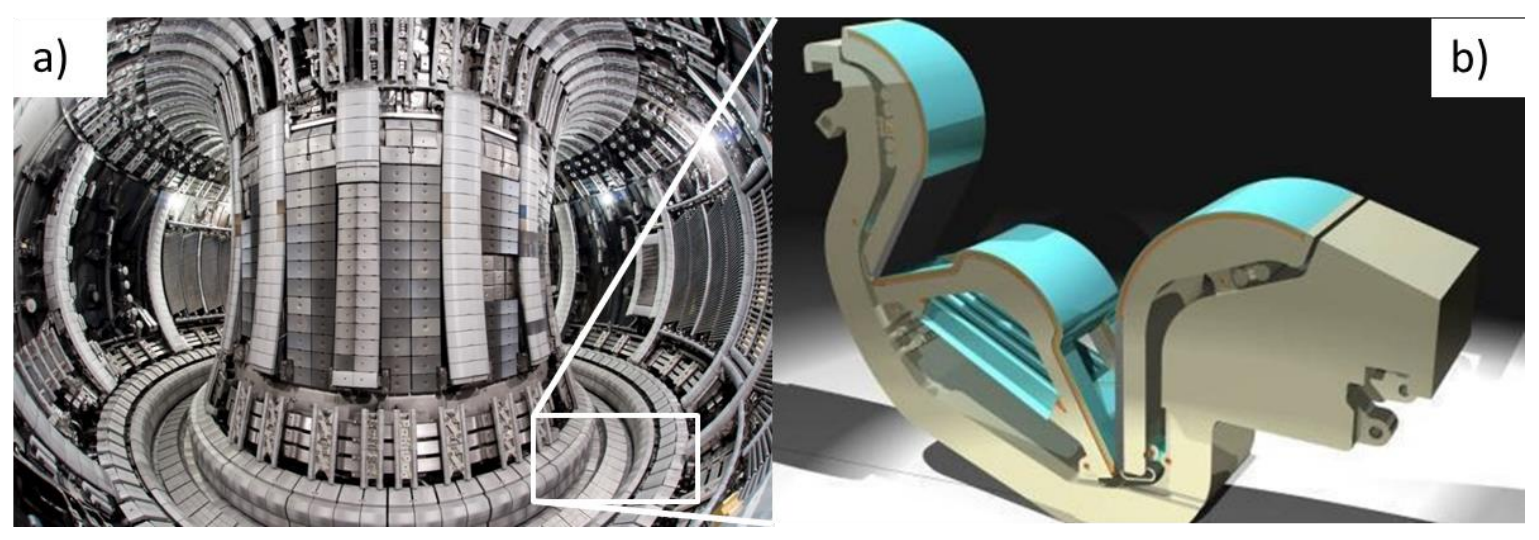

Figure 1. a) Image of the JET reactor with the divertor section highlighted in the box and b) design of a single cassette used in the divertor structure (reproduced from Eurofusion)

During service, the divertor will be exposed to high temperatures (up to $1400^{\circ} \mathrm{C}$ ) in addition to the aforementioned neutron radiation damage and implantation of low energy $(10 \mathrm{seV}) \mathrm{He}$ ions from the fusion reaction. In addition to this, He will be produced from $(n, \alpha)$ transmutation reactions in the bulk of the divertor structure which will combine with radiation induced defects, leading to a complex damage process. Thus, a concerted effort is underway to study the behaviour of $\mathrm{W}$ under the anticipated extreme environments, employing a range of irradiation conditions such as projectile, ion energy, irradiation temperature and damage dose. This article reviews data from neutron and ionirradiations of $\mathrm{W}$, comparing the damage features and property changes observed with the chosen experimental technique.

\section{Irradiation techniques}

Radiation damage causes the displacement of atoms from their lattice sites in a material, creating knock-on atoms which then go on to cause further damage. This is known as the damage cascade. These displaced atoms (which come to rest in interstitial positions) may either recombine with a vacant site, or, they may combine with another interstitial and grow into a larger defect. Vacancy type defects may also grow in the same manner or form directly from the collapse of the cascade 
itself. Collectively, these processes produce defect features such as dislocations lines, loops and cavities. These defects can cause the material to swell, become brittle or harden which may lead to structural failure as well as degradation of the materials thermophysical and thermomechanical properties, reducing the efficiency and integrity of the component.

Another issue arising from neutron irradiation is the creation of transmutation products which can further degrade the material's mechanical and thermophysical properties via second phase precipitation and cavity nucleation which is aided by the generation of $\mathrm{H}$ and $\mathrm{He}$ gases from $(\mathrm{n}, \mathrm{p})$ and $(n, \alpha)$ reactions, respectively. Figure 2 shows the concentration of transmutation products formed when $\mathrm{W}$ is exposed to a $1.6 \mathrm{GW}$ DEMO reactor fusion neutron spectrum for two full power years [3], it shows that the main transmutation products are Re, Ta and Os, with around 6 and 2 appm of $\mathrm{H}$ and $\mathrm{He}$ gas being produced per year, respectively. However, the rate of transmutation varies 
depending on power output and neutron flux of the DEMO reactor concept (see further discussion in Ref. [4]).

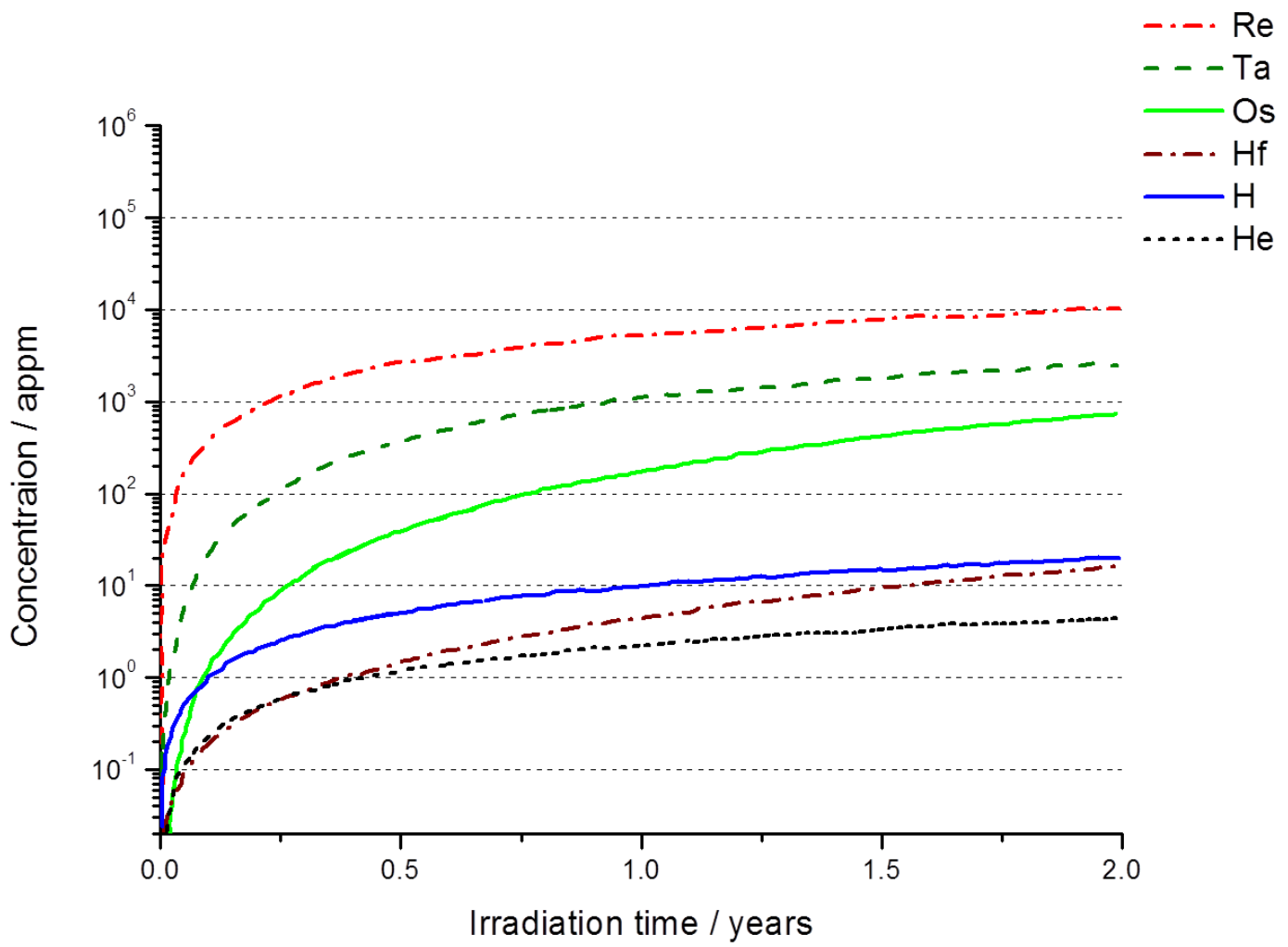

Figure 2. Graph showing transmutation products of $\mathrm{W}$ under a $1.6 \mathrm{GW}$ DEMO fusion reactor (neutron spectra $5 \times 10^{14} \mathrm{n} \mathrm{cm}^{-2} \mathrm{~s}^{-1}$ ) for two full power years, reproduced from Ref. [3]

Employing both thermal and fast fission neutrons ( $<0.1 \mathrm{MeV}$ and $>0.1 \mathrm{MeV}$, respectively) to study the irradiation behaviour of nuclear materials yields the closest representation to the fusion neutron (14.1 MeV) environment the material will be exposed to. However, existing neutron irradiation facilities can still not achieve the high energy fusion neutron spectra which will affect transmutation pathways due to the different neutron-capture cross sections of $\mathrm{W}$ under varying neutron spectra. In addition, experimental campaigns can be expensive, firstly as it is necessary to gain access to the small number of facilities available and second due to potential problems in the handling of materials after irradiation and/or storage of irradiated samples until the activity has decayed enough for sample manipulation. Furthermore, if one wants to study higher damage doses up to the anticipated end-of- 
life dose of the material after neutron irradiation the experiments become highly time-consuming due to slow damage rates.

Ion beam irradiation can serve as a good proxy for neutron damage as the irradiating ion causes atomic displacements of the $\mathrm{W}$ lattice. Access to ion-irradiation facilities is much more readily available and, as the irradiated specimens are generally not activated after irradiation, the requirement for expensive handling facilities is avoided. Ion irradiation can also produce the anticipated high damage levels anticipated from the fusion reactor core in much shorter times $(10 \mathrm{~s}$ of hours) with the use of self or heavy ion irradiation to impart displacement damage, making it cost and time effective in comparison to neutron irradiations.

However, the experiments need to be designed carefully, with due consideration of the projectile used, dose rate and amount of ion implantation caused, as this can affect the types of damage features observed. For example, a self-ion irradiation will implant more interstitials than would be present in a neutron irradiation, which may reduce the observation of vacancy type defects (vacancy-type dislocation loops and voids) via recombination or higher frequency of interstitial-type defects. In addition to this, the high dose rates $\left(10^{-3} \mathrm{DPA} / \mathrm{s}\right)$ that make ion beam irradiations attractive may hinder comparison with neutron irradiated materials which have much lower dose rates $\left(10^{-7} \mathrm{DPA} / \mathrm{s}\right)$ [5]. Mansur [6,7] proposed a temperature shift for high dose rate ion experiments whereby the irradiation temperature is increased as to achieve comparable rates of defect arrival at sinks in the short time frame of an ion irradiation as compared to the longer time scales of neutron irradiations. Packan et al. [8] irradiated Ni samples to 0.9 DPA $\left(10^{-7} \mathrm{DPA} / \mathrm{s}\right)$ under fast neutron irradiation at the Oak Ridge Research Reactor (ORR) $(\mathrm{E}>0.1 \mathrm{MeV})$ at temperatures between 300 and $850^{\circ} \mathrm{C}$ showing that to achieve a similar rate of swelling the irradiation with $4.0 \mathrm{MeV} \mathrm{Ni}$ ions to $1.0 \mathrm{DPA}$ $\left(10^{-3} \mathrm{DPA} / \mathrm{s}\right)$ required a temperature shift of $\sim 200^{\circ} \mathrm{C}$. However, this temperature shift is predicted to vary for emulation of differing types of microstructural defect such as void growth regimes, precipitation rates and dislocation loop sizes and populations due to ranges in activation energies for defect mobility (such as interstitials, vacancy and solute migration energies). Thus, Zinkle and Snead 
[9] suggest that a single temperature shift cannot achieve a complete microstructural correlation between ion and neutron irradiations performed at different dose rates. This is due to altering of defect arrival rates at sinks, i.e. increasing irradiation temperature in an ion beam experiment to capture vacancy arrival at voids over the longer time scales of a neutron irradiation and emulate swelling rate behaviour may inadvertently lead to increased dislocation loop mobility resulting in differences in the dislocation microstructure. Further to this, rastering of the ion beam can increase the dose rate by $\sim 40$, yielding a very high defect production rate during the time the beam illuminates the area, However, subsequent defect annealing may then occur in the remaining time of the raster cycle [5], and care should be taken when comparing results from rastered and static ion beam irradiations.

Surface effects also need to be taken into consideration when performing ion irradiation experiments as these will have consequences on the radiation damage features observed. Zinkle and Snead [9] discuss the effects of the loss of point defect supersaturation at planar sinks such as free surfaces and grain boundaries. This will, for example, lead to changes in relative defect populations such as interstitial and vacancy dislocation loop frequencies, with the prior being highly mobile to free surfaces and sinks. This has been reported to result in peak swelling zones closer to free surfaces [10-12] attributed to the accelerated loss of interstitials and interstitial clusters, causing a supersaturation of vacancies and increase in void swelling. Zinkle and Snead [9] also propose that the irradiated microstructure should be characterised as a function of depth as to determine 'safe zones' for analysis, considering effects from both the free surface and from the ion implantation zone. Doyle et al. [13] examined the size of these 'safe zones' in self ion irradiated Fe as a function of ion energy by modelling radiation enhanced diffusion (RED) of ion implanted profiles from the Stopping Range of Ions in Matter (SRIM) [14] Monte Carlo simulations with Fick's second law using experimentally determined RED coefficients. The authors report that in the current effort to emulate high DPA, high temperature radiation for fusion reactors (above vacancy mobility activation temperatures for most metals), void denuded regions near the surface arise from loss of local vacancy supersaturation via diffusion to the free surface along with void suppression in the ion implantation 
peak. The authors reported that for a low assumed RED coefficients $\left(10^{-20}\right.$ to $\left.10^{-19} \mathrm{~m}^{2} / \mathrm{s}\right)$ broadening of the ion implanted layer was very small and a suitable safe zone for analysis could be achieved for 5-6 MeV self-ions into Fe. However, at higher RED coefficients of $\sim 10^{-19}$ to $10^{-18} \mathrm{~m}^{2} / \mathrm{s}$, significant peak broadening of the ion implanted layer occurs and no valid safe zone exists below $\sim 6-8 \mathrm{MeV}$ self-ion energies. Similar coupling of SRIM with RED coefficients for the W system would aid the design of the ion irradiation parameters to give suitable regions for analysis.

Post irradiation characterisation of ion beam irradiated materials must also consider the range of damage and implantation profiles caused as the impinging ion comes to rest within the material. This can lead to variations in results depending on the depth and scale at which one performs the measurement or characterisation. Equally, thin-film analysis techniques such as glancing incident Xray diffraction, nanoindentation and 2-D time domain thermoreflectance must be employed to characterise the irradiated layer.

Although bulk, ex-situ ion irradiations provide useful information when examining physical properties of materials, these studies intrinsically suffer from the fact it is only possible to characterise the 'end point'. In-situ ion irradiation of transmission electron microscope (TEM) foils provides a highly detailed, real time analysis of the developing defect population during irradiation [15]. Coupled with TEM techniques, such as electron diffraction, dark-field fielding imaging and chemical analysis between irradiation steps a highly detailed analysis of defect population (e.g. dislocation loop type and nature) and change in chemical properties (such as radiation induced segregation) as a function of damage dose may be developed. However, an issue of the in-situ irradiation of thin films is the proximity of the two surfaces of the TEM foil which will act as large sinks for migrating defects, potentially altering the defect population (i.e. dislocation loop density, interstitial vs. vacancy type dislocation loops) compared to bulk ion irradiation experiments which will be discussed throughout this review. For example, Fikar et al. [16] used atomistic simulations (combining three embedded atom method with simple elastic solutions) to study $\boldsymbol{b}= \pm 1 / 2<111>$ dislocation loop mobility to free surfaces in W. The authors found that $\boldsymbol{b}= \pm 1 / 2<111>$ loops, $\sim 7.5 \mathrm{~nm}$ 
in diameter may escape from a depth of $13.9 \mathrm{~nm}$ from the sample surface. Thus, for a $55 \mathrm{~nm}$ TEM foil this would mean $\sim 50 \%$ of the loops are lost to surface annihilation leading to only around half of the nucleated loops being observed by in-situ ion irradiation and TEM.

Where other reviews $[17,18]$ have aimed at collating the behaviour of $\mathrm{W}$ under irradiation conditions to comment on its suitability as use as a structural nuclear material, this paper aims to critically review how well the state of the art ion irradiation experiments can emulate fundamental radiation damage mechanisms and processes in $\mathrm{W}$. This review will first describe the nucleation mechanisms of dislocation loop types in body centred cubic (BCC) metals, which remain unclear. The general microstructural damage features and defect types observed in neutron, heavy ion and light ion irradiated W will then be discussed. Differences in the damage microstructures will then be reviewed and attributed to the fundamental atomistic mechanisms at play in each irradiation scenario before comparison of property changes (mechanical and thermal properties) of $\mathrm{W}$ under neutron and ion irradiation.

\section{Dislocation loop nucleation in BCC metals}

The types of dislocation loops arising under irradiation can play and important role in the degradation of the materials mechanical properties. For example, dislocation loops with $\boldsymbol{b}= \pm 1 / 2<111\rangle$ are generally glissile whereas those with $\boldsymbol{b}=\langle 100\rangle$ are sessile for BCC materials [19-21] and so the former may more readily migrate to sinks (such as grain boundaries or annihilate with other defects), however, the latter, being immobile will accumulate in the microstructure. This would create a larger number of immobile pinning points thus increasing the radiation induced hardening and embrittlement of the material.

Self-interstitial atoms (SIAs) in the BCC lattice may occupy tetrahedral or octahedral sites, however, this simple model of SIAs is not a true physical picture of the stable configuration in BCC metals [5]. The lattice will actually adopt a 'split-interstitial' configuration and share a single lattice site with the SIA. In addition the SIA may also adopt a crowdion configuration where three atoms share 
two lattice sites. The atom nuclei repel each other and so atoms will arrange themselves in the lowest energy configuration corresponding to crystallographic orientations. Figure 3 shows the different orientations the split-interstitial may adopt, the lowest energy configuration for the BCC lattice is for the SIA to adopt the $<110>$ direction [5]. However, formation energies of each SIA defect orientation in $\mathrm{W}$ and type are shown in Table 1 from Ref. [22] showing the $<111>$ dumbbell is the preferred configuration.

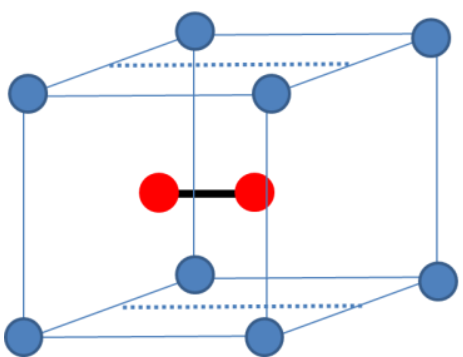

$<100>$ dumbbell

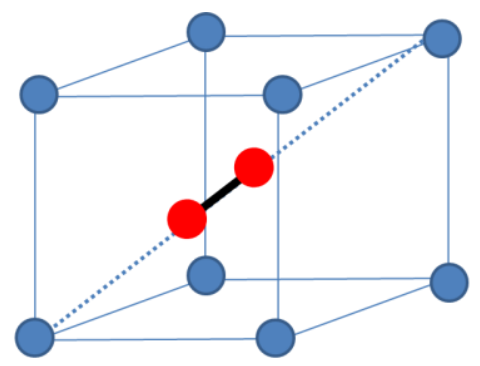

$<111>$ dumbbell

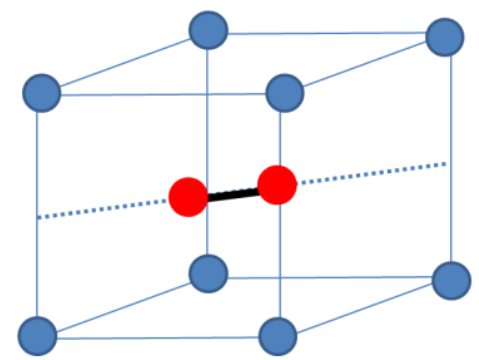

$<110>$ dumbbel

Figure 3. Figure showing the different crystallographic orientations of SIAs adopting the splitinterstitial model in the BCC unit cell.

\begin{tabular}{|c|c|}
\hline Defect type & Formation energy/ eV \\
\hline$<111>$ dumbbell & 9.548 \\
\hline$<111>$ crowdion & 9.551 \\
\hline$<110>$ dumbbell & 9.844 \\
\hline$<100>$ dumbbell & 11.49 \\
\hline Octahedral SIA & 11.68 \\
\hline Tetrahedral SIA & 11.05 \\
\hline
\end{tabular}

Table 1. Formation energies of SIA defects calculated for $\mathbf{W}$ from Ref. [22]

Two types of interstitial loops were found to be present in irradiated BCC Fe alloys, and were of $\boldsymbol{b}$ $=1 / 2<111>$ and $\boldsymbol{b}=\langle 100\rangle$ type as first reported by Eyre and Bullough [23]. They concluded that the dislocation loop nucleus was a faulted interstitial-type dislocation loop on $\{110\}$ planes as predicted 
from the theoretical split-interstitial model [5]. Addition of further interstitials produces a platelet of split-interstitials on a $\{110\}$ type plane. This dislocation core contains a high energy stacking fault in the $\{110\}$ stacking sequence and Eyre and Bullough [23] propose this would be removed by shear produced from the partial dislocation reactions shown in Eq.1 and Eq. 2 [23];

$$
\begin{array}{ll}
\frac{1}{2}[110]+\frac{1}{2}[00 \overline{1}] \rightarrow \frac{1}{2}[11 \overline{1}] \quad \text { Eq. } 1 \\
\frac{1}{2}[110]+\frac{1}{2}[\overline{1} 10] \rightarrow[010] \quad \text { Eq. } 2
\end{array}
$$

Unfaulting of a $1 / 2<110>$ loop via Eq. 2 has been shown to have a probability of 1 in $10^{19}$ in Mo at room temperature [24]. Jäger and Wikens [25] observed the formation of a small fraction of faulted $\boldsymbol{b}= \pm 1 / 2<110\rangle$ loops in $\mathrm{W}$ with $60 \mathrm{keV}$ Au ions. The authors stated that if the loops of this type reached a critical radius, between $0.9-2.0 \mathrm{~nm}$ then the loop would shear to remove the stacking fault as described by Eyre and Bullough [23]. However, Jäger and Wikens [25] did not observe any $\boldsymbol{b}=$ $<100>$ type dislocation loops (although they stated around $10 \%$ of loops were not analysed) and a dominance for $\boldsymbol{b}=1 / 2<111>$ types, agreeing with the very low probability of a faulted loop shearing via Eq. 2.

However, Masters proposed that in BCC Fe <100> type loops can form by the interaction of two mobile $\boldsymbol{b}= \pm 1 / 2<111>$ loops, as shown in Eq. 3 from Ref. [26]

$$
\frac{1}{2}[111]+\frac{1}{2}[\overline{1} \overline{1} 1] \rightarrow[001] \quad \text { Eq. } 3
$$

This has been observed experimentally, for example in $\mathrm{FeCr}$ alloys by Prokhodtseva et al. [21]. The authors irradiated $\mathrm{Fe}$ and $\mathrm{FeCr}$ alloys in single beam experiments employing $500 \mathrm{keV}$ Fe ions and also in simultaneous dual beam experiments using the same energy Fe ions with co-implantation of $10 \mathrm{keV}$ He ions. The authors [21] noted a shift in the type of Burgers vectors of the dislocation loop population, from a dominance of $\boldsymbol{b}=\langle 100\rangle$ type in the single beam Fe ion irradiation to a dominance of $\boldsymbol{b}= \pm 1 / 2<111>$ loops in the simultaneous $\mathrm{Fe}$ and He ion irradiations. Thus, for the $\mathrm{FeCr}$ alloys, 
Prokhodtseva et al. [21] proposed a mechanism whereby in the presence of He, the mobility of the $\boldsymbol{b}$ $=\langle 111>$ type loops is low, leading to less chance of interaction and coalescence with another $\boldsymbol{b}=$ $<111>$ type loop that would lead to loops with $\boldsymbol{b}=\langle 001\rangle$.

The results of Prokhodtseva et al. [21] were in good agreement with modelling work of Xu et al. [19] who used atomistic kinetic Monte Carlo (kMC) simulations to show that the formation of $<100\rangle$ loops occurs directly from atomistic interaction between two $\boldsymbol{b}=1 / 2<111>$ loops in Fe.

However, Sand et al. [27] have performed molecular dynamics (MD) simulations on the impact of a $150 \mathrm{keV}$ W primary knock-on atom (PKA) and found that the formation of $\boldsymbol{b}=\langle 100\rangle$ loops occurred as a direct result of the cascade, where the high energy density of the cascade would provide a higher probably of a loop unfaulting via Eq. 2. English and Jenkins [24] also reported that the number of $\boldsymbol{b}$ $=<100>$ loops increased in Mo irradiated with $\mathrm{Sb}^{+}, \mathrm{Sb}_{2}{ }^{+}$and $\mathrm{Sb}_{3}{ }^{+}$molecular ions with both ion mass and energy and attributed this to the increased average energy density of the cascade and extended thermal spike lifetime, leading to an increased likelihood of loop unfaulting according to Eq. 2.

The effects of PKA spectrum, C impurities and Re transmutation on interstitial loop dislocation nucleation has been modelled by Castin et al. [28] using objective (o)kMC simulations. The authors reported that under neutron relevant PKA spectra $(\sim 100 \mathrm{keV})$ large interstitial loop dislocations visible in a TEM ( $>1 \mathrm{~nm})$ are rarely produced directly in the cascade and form from the coalescence of 3-D migrating mono-SIAs and 1-D migrating SIA clusters with Re aiding their nucleation and growth by reducing 1-D SIA migration lengths. However, in high energy ( 2 and $18 \mathrm{MeV}$ ) W ion irradiations where the PKA spectra increases by two order of magnitude, dislocations loops visible in a TEM form directly from the collision cascade. The authors [28] comment that as the SIA loops are fast migrating 1-D defects a $\mathrm{C}$ impurity level of around $10 \mathrm{appm}$ is required to provide trapping and defect survival as to achieve parity with experimentally obtained TEM measurements. The observation of decreased SIA mean free path with increased Re content by Castin et al. [28] agreed well with kMC simulations by Bonny et al. [29] who found that addition of 0.1 at $\%$ Re to the W was significant enough to decrease SIA cluster mobility in 1-D. However, unlike Castin et al. [28] the 
authors [29] state this could lead to increased SIA-vacancy recombination, as Frenkel Pairs (FPs) would remain in proximity and so lead to fewer SIA defect clusters nucleating and reduced void swelling.

This has shown there are important considerations when aiming to study dislocation loop nucleation mechanisms and resulting dislocation structures (i.e. Burgers vectors and loop nature) when comparing neutron irradiations (PKA energies limited to below $\sim 400 \mathrm{keV}$ and interplay with transmutation products) with ion beam irradiations (maximum PKA energies may be $>\mathrm{MeV}$ and trapping effects from $\mathrm{C}$ impurities). With these dislocation loop type nucleation mechanisms now discussed, and the varied empirical results for BCC metals highlighted, the types of loop observed in neutron and ion irradiated $\mathrm{W}$ will now be discussed.

\section{Microstructural damage features}

\subsection{Neutron irradiation}

Recent neutron irradiation experiments have examined the effect of neutron dose, spectra and irradiation temperature on the damage microstructures of $\mathrm{W}$ and $\mathrm{W}$ based alloys using the High Flux Isotope Reactor (HFIR, USA) as well as the Japanese Materials Test Reactor (JMTR, Japan) and the test sodium-cooled fast reactor, Joyo (Japan) [30-34]. A summary of TEM micrographs are shown in Figure 4 depicted in a matrix of irradiation temperature and DPA [30-35] showing that at temperatures $<800^{\circ} \mathrm{C}$ and low dose $(\sim 1.5 \mathrm{DPA})$ the microstructures appear to be a mixture of dislocation loops and voids, however, at higher temperatures and doses these become dominated by voids.

Characterisation of dislocations and voids in neutron irradiated $\mathrm{W}$ samples under both thermal and fast neutron spectra (HFIR and Joyo reactors) to low doses ( $<1.0$ DPA) at temperatures up to $800^{\circ} \mathrm{C}$ has been performed by Hasegawa et al. [30,36]. At temperatures $<750^{\circ} \mathrm{C}$, the damage microstructure was found to be a mixture of dislocation loops $(<10 \mathrm{~nm})$ and small $(<10 \mathrm{~nm})$ voids. At higher temperatures $\left(>750^{\circ} \mathrm{C}\right)$ and higher doses $(\sim 1.5 \mathrm{DPA})$ the damage microstructure became dominated 
by voids which were larger in size $(\sim 10 \mathrm{~nm})$. Fukuda et al. [32] also irradiated $\mathrm{W}$ and W-Re alloys in HFIR to 1.0 DPA at 500 and $800^{\circ} \mathrm{C}$. The authors [32] noted at the lower irradiation temperature of $500^{\circ} \mathrm{C}$, the damage microstructure consisted of needle-like, intermetallic Re precipitates which were found to be $\sigma\left(\mathrm{W}_{7} \mathrm{Re}_{6}\right)$ and $\chi\left(\mathrm{WRe}_{3}\right)$ phases from electron diffraction. Under equilibrium conditions these phases should only form at higher temperatures $\left(>500^{\circ} \mathrm{C}\right)$ and Re concentrations [37], however, under highly non-equilibrium conditions of neutron radiation, these phases have been observed to form [32] at Re concentrations lower than the solubility limit which will be discussed further in $\S 5.1$. Dislocation loops $\sim 3 \mathrm{~nm}$ in diameter with a density of $3.3 \times 10^{22} \mathrm{loops} / \mathrm{m}^{3}$ were found at $500^{\circ} \mathrm{C}$ and at $800^{\circ} \mathrm{C}$ the authors [32] observed voids of $\sim 4 \mathrm{~nm}$ diameter with a volumetric density of $8.0 \times 10^{21}$ voids $/ \mathrm{m}^{3}$ with no dislocation loops reported.

A void lattice was found to form in neutron irradiated $\mathrm{W}$ in the Joyo reactor at an irradiation temperature of $750^{\circ} \mathrm{C}$ at $1.5 \mathrm{DPA}$ by Tanno et al. [33,34]. Voids of $\sim 5 \mathrm{~nm}$ diameter were found to form in all samples irradiated at 400 and $750^{\circ} \mathrm{C}$ in contrast to the work of Fukuda et al. [32] who only observed void formation at higher temperature $\left(800^{\circ} \mathrm{C}\right)$. However, the work by Fukuda et al. [32] was performed in HFIR under thermal neutron irradiation which may be the cause of the differences in void nucleation temperature compared with the work of Tanno et al $[33,34]$. Tungsten has a higher absorption cross section for thermal neutrons leading to increased transmutation precipitation [38] which may hinder vacancy mobility at these temperatures via a solute-vacancy trapping mechanism. This has previously been shown to suppress void nucleation [39] and may have led to no observable voids in the HFIR experiments at lower temperatures [32]. Needle-like precipitates were also observed to form which were attributed to $\chi$ phase and were aligned with $\{110\}$ planes. A void lattice has also been reported by Sikka and Moeteff [35] who exposed W to around 1.0 DPA (fluence of $\sim 10^{22} \mathrm{n} . \mathrm{cm}^{-2}$ ) in the Experimental Breeder Reactor (EBR-II, USA) at $550^{\circ} \mathrm{C}$. These lattices will be discussed further in $\S 4.3$ and 4.5.

Unfortunately, recent characterisation of neutron irradiated $\mathrm{W}$ has predominantly focused on dislocation loop, void and precipitate size, with no characterisation of dislocation type being 
performed $[31,33,34,40,41]$ unlike the ion irradiation experiments which will be discussed in the following section. Rau [42] annealed fast-neutron irradiated $\left(E>1 \mathrm{MeV}, \sim 10^{21}\right.$ n.cm $\left.{ }^{-2}\right) \mathrm{W}$ to a temperature of $1100^{\circ} \mathrm{C}$ for $315 \mathrm{~h}$ and reported vacancy dislocation loops with $\boldsymbol{b}= \pm 1 / 2<111>$ type only. However, no dislocation analysis is reported pre-annealing and so it is not clear whether loops with $\boldsymbol{b}=\langle 100\rangle$ were present prior to annealing. However, Rau et al. [43] also performed TEM analysis of fast-neutron irradiated Mo at $700^{\circ} \mathrm{C}$ noting a dominance of $\boldsymbol{b}= \pm 1 / 2<111>$ and $\sim 10 \%$ of $\boldsymbol{b}=\langle 100\rangle$ type loops. This work will be discussed here as to permit further comparison between dislocation loop nucleation mechanisms in ion and neutron irradiations in $\mathrm{BCC}$ metals in the following sections.

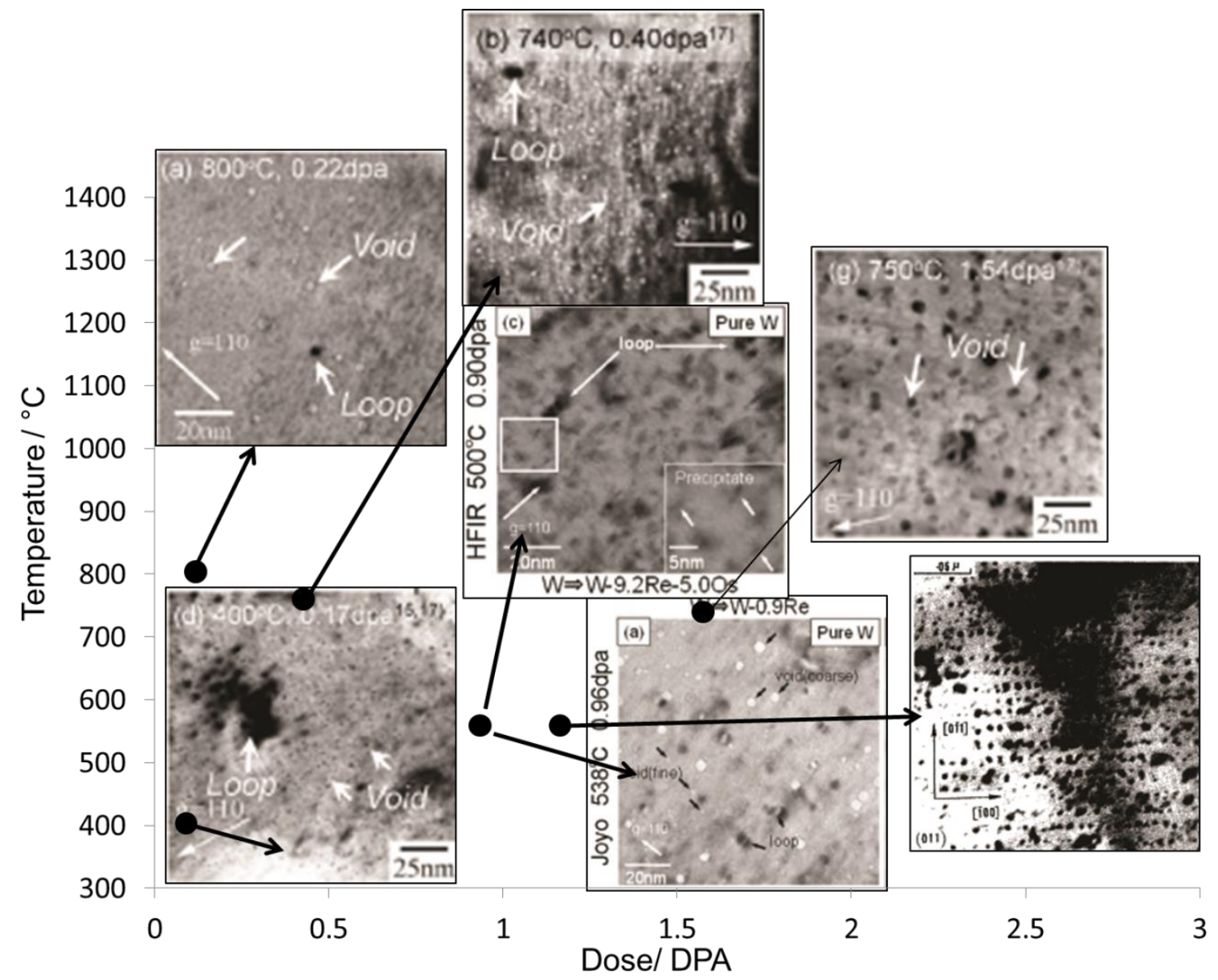

Figure 4. Summary of micrographs from neutron irradiated $\mathrm{W}$ represented a function of the irradiation temperature and DPA reproduced from Refs. [30-35] 


\subsection{Heavy ion irradiation}

The presence of a majority of dislocation loops had $\boldsymbol{b}= \pm 1 / 2\langle 111\rangle$ and a minority of $\boldsymbol{b}= \pm 1 / 2<110\rangle$ has been reported in $\mathrm{W}$ irradiated with $60 \mathrm{keV} \mathrm{Au}$ ions at room temperature by Jäger and Wikens [25]. In the work [25] report that all the dislocations were of vacancy type and formed in the cascade core via cascade collapse, although it has been reported that the majority of dislocation loops formed under irradiating conditions are of the interstitial type in BCC metals [23].

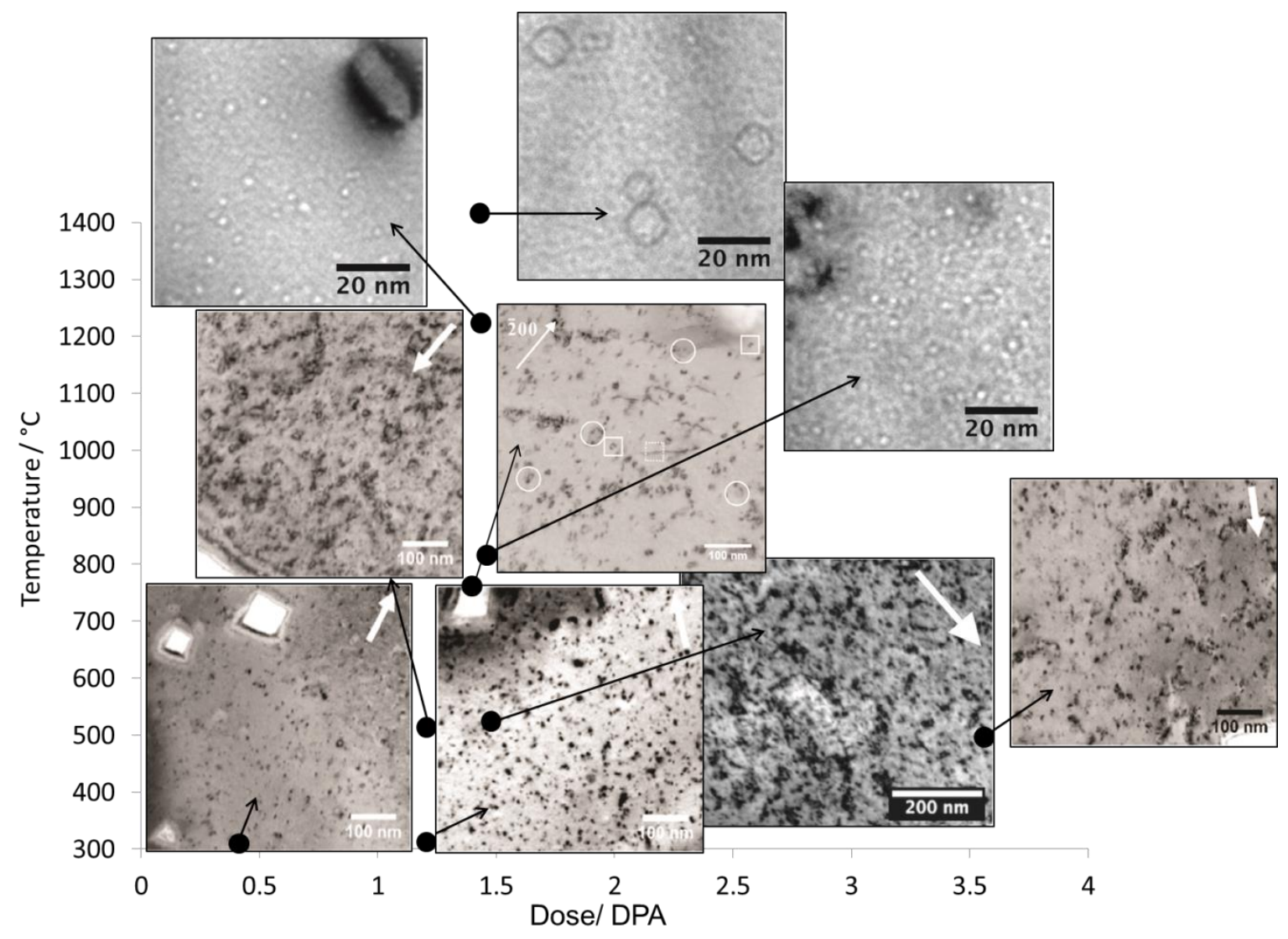

Figure 5. Summary of micrographs from self-ion irradiated $\mathbf{W}$ and in-situ annealing experiments represented a function of the irradiation temperature and DPA reproduced from Refs. $[20,44-$ 46]

Figure 5 show a summary of the TEM damage microstructures from both ex-situ and in-situ $\mathrm{W}^{+}$ion irradiated samples and post irradiation in-situ annealed samples as a function of temperature and dose from Refs. [20,44-46] and Figure 6 and Figure 7 compare the dislocation sizes and volumetric 
densities reported in $\mathrm{W}$ under neutron $[32,36,41,47]$ and ion irradiations $[20,46,48,49]$. The pioneering work of Yi et al. $[20,44,45]$ examined the effects of self-ion irradiation on the damage microstructure of $\mathrm{W}$ using $150 \mathrm{keV}$ or $2 \mathrm{MeV} \mathrm{W}^{+}$during in-situ and ex-situ experiments respectively, to a dose of 3 DPA at temperatures up to $800^{\circ} \mathrm{C}$. At all irradiation temperatures the authors reported that the defect microstructure consisted of loops with no void formation, unlike neutron irradiations. Dislocation loops in the ex-situ experiments had a diameter of $\sim 1-2 \mathrm{~nm}$ and volumetric densities were reported to be $\sim 4 \times 10^{22}$ loops $/ \mathrm{m}^{3}$ at $1.2 \mathrm{DPA}$ and an irradiation temperature of $500^{\circ} \mathrm{C}$ [20] agreeing well the neutron irradiation work of Fukuda et al. [32] as seen in Figure 6 and Figure 7 (3.3 $\times 10^{22}$ loops $/ \mathrm{m}^{3}$ at $1.0 \mathrm{DPA}$ at $\left.500^{\circ} \mathrm{C}\right)$. Dislocation loops in the in-situ case were similar in size $(\sim 1-$ $2 \mathrm{~nm})$ at the same irradiation dose and temperature $\left(1.0 \mathrm{DPA}\right.$ and $\left.500^{\circ} \mathrm{C}\right)$ but higher in volumetric density with $\sim 8 \times 10^{22} \mathrm{loops} / \mathrm{m}^{3}$. This may be due to the peak damage layer of $150 \mathrm{keV} \mathrm{W}$ ions being $\sim 15 \mathrm{~nm}$ into the foil [45] giving rise to a highly dense concentration of damage as compared with the ex-situ case, or that the ex-situ experiments were performed with a rastered ion beam [20], potentially leading to defect annealing during the remaining rastering cycle. Equally there was no formation of a void lattice under the same conditions (irradiation temperature of $500^{\circ} \mathrm{C}$ ) as the neutron irradiations which may be due to lack of gas impurity atoms or implantation of interstitial ions supressing void formation. Heavy ion irradiation of Mo with Ta ions has produced void lattices, however, gas impurity atoms were reported to aid their formation and alignment [50]. 


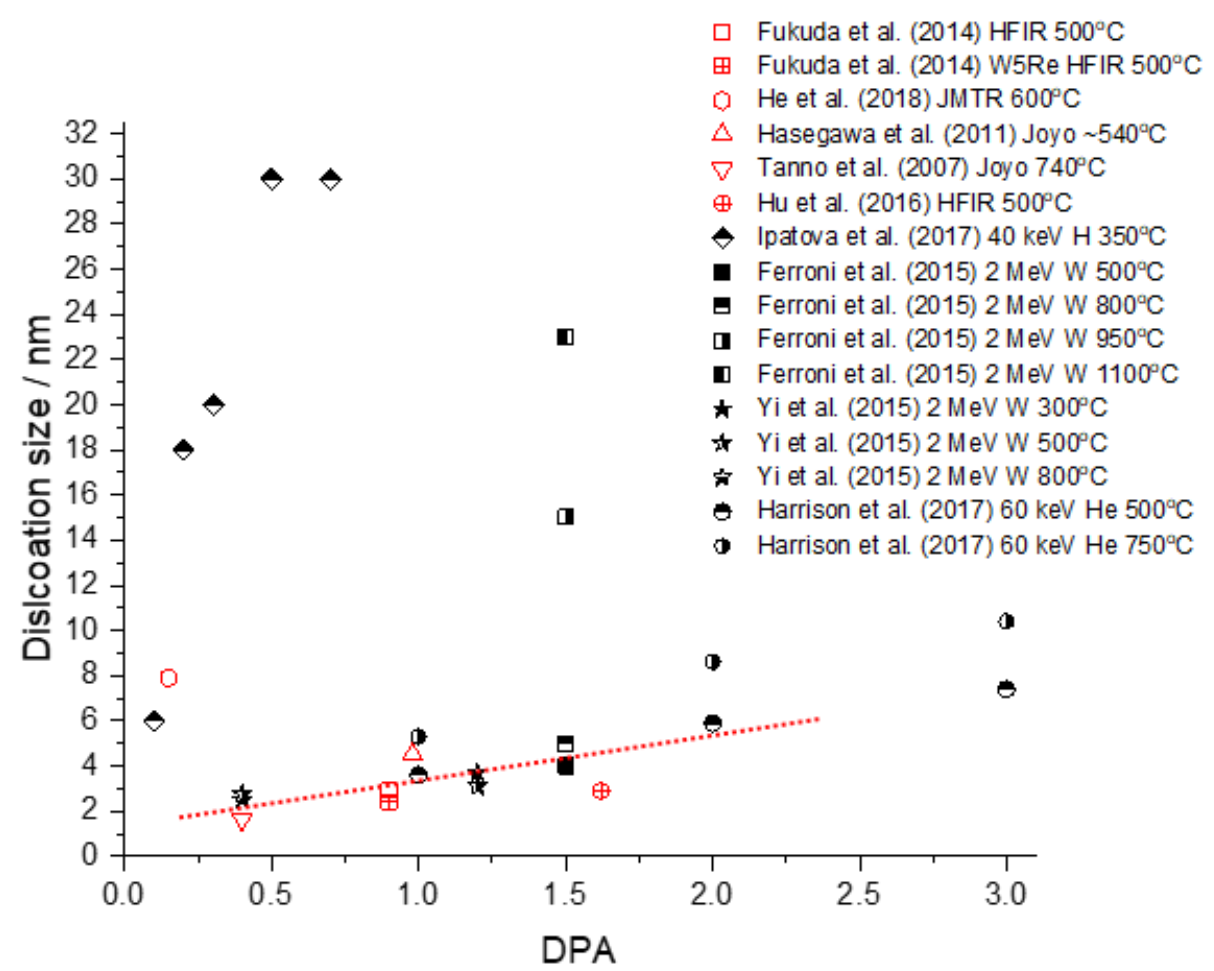

Figure 7. Plot of dislocation loop sizes for neutron irradiated $W$ and WRe alloys (open red symbols, line to guide eyes for neutron data only) $[32,36,41,47]$ and ion irradiated $W$ samples (semi/closed black symbols) $[20,46,48,49]$.

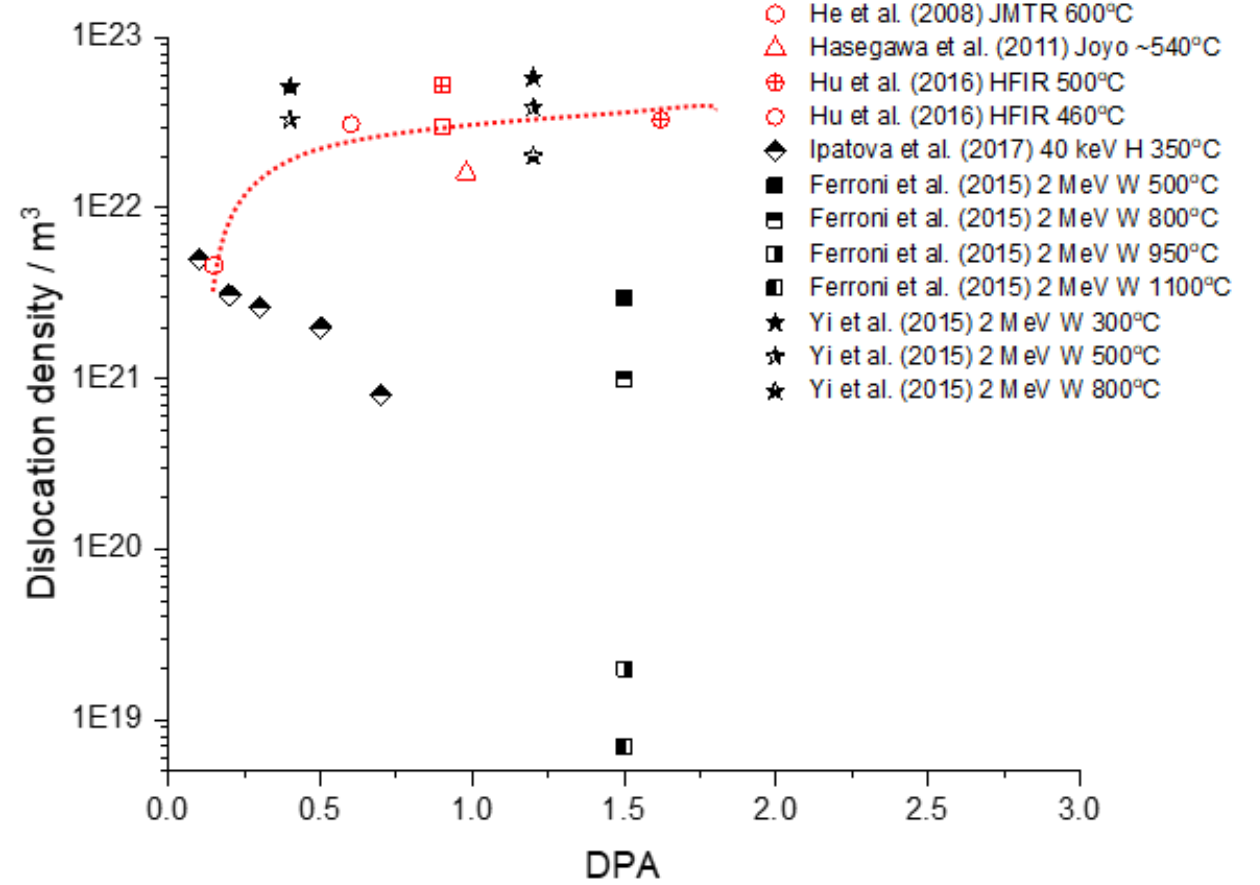

Figure 6. Plot of dislocation loop densities for neutron irradiated $W$ and WRe alloys (open red symbols, line to guide eyes for neutron data only) $[32,36,41,47]$ and ion irradiated W samples (semi/closed black symbols) [20,46,48]. 
Small dislocation loops of both vacancy and interstitial types we observed by Yi et al. $[20,44,45]$. The loops mainly had a Burgers vector of $\boldsymbol{b}= \pm 1 / 2<111>$ but a minority with $\boldsymbol{b}= \pm 1 / 2<100\rangle$ (dislocation loop type will be discussed further in $\$ 4.4$ ) were found. The authors $[20,45]$ found the fraction of $\boldsymbol{b}=\langle 100\rangle$ type loops decreased with increasing irradiation temperature which is attributed to these type of dislocation loops more rapidly becoming unstable as a function of size as compared with the $\boldsymbol{b}= \pm 1 / 2<111\rangle$ type due to their free energies [22]. The $2 \mathrm{MeV} \mathrm{W}^{+}$ex-situ irradiations will have resulted in the peak damage layer being $~ 100 \mathrm{~nm}$ from the sample surface and so surface effects cannot be negated in either the ex-situ or in-situ case. However, accelerated loss of glissile $\boldsymbol{b}= \pm 1 / 2<111>$ dislocation loops to the free surfaces as irradiation temperature was increased would have yielded an increase in the fraction of sessile $\boldsymbol{b}=\langle 100\rangle$ type loops and so the observation of an increase in the $\boldsymbol{b}= \pm 1 / 2<111>$ loop population is consistent with an decreasing stability of $\boldsymbol{b}=<100>$ type loops as they grow in size with increasing irradiation temperature.

The observation of interstitial loops were attributed to the nucleation and growth of interstitial clusters as all irradiation temperatures were above the thermal activation energy for mono-interstitial migration and interstitial release from traps (e.g. immobile vacancies, grain boundaries or surfaces) which occurs at temperatures above $-173^{\circ} \mathrm{C}$, and this is referred to as stage II recovery [46]. The vacancy type loops observed by Yi et al. $[20,44,45]$ were attributed to direct formation from cascade collapse, similar to the nucleation mechanism reported by Jäger and Wikens [25] and loop growth would occur via absorption of monovacancies, which occurs at $\sim 350^{\circ} \mathrm{C}$, with an activation energy of $1.7 \mathrm{eV}[46,51]$ (stage III recovery mechanism). Stage IV recovery occurs at temperatures above $\sim 720^{\circ} \mathrm{C}$ and has been attributed to the migration of vacancy-impurity complexes. Stage $\mathrm{V}\left(\sim 870^{\circ} \mathrm{C}\right)$ is attributed to the migration of larger vacancy clusters [46]. In the in-situ self-ion irradiations $[44,45]$ the fraction of interstitial type loops increased as a function of irradiation temperature at a dose of 1.0 DPA from $\sim 50 \%$ at $500^{\circ} \mathrm{C}$ to $70 \%$ at $800^{\circ} \mathrm{C}$, whereas in the ex-situ irradiations [20], interstitial loop population increased from $\sim 75 \%$ at $300^{\circ} \mathrm{C}$ to $\sim 95 \%$ at $500^{\circ} \mathrm{C}$ (at $1.2 \mathrm{DPA}$ ). This lower interstitial loop population in the in-situ case may be attributed to accelerated loss of interstitial-type 
loops and interstitial clusters to the TEM foil free surfaces, which is reduced in the near-surface exsitu irradiations.

Ferroni et al. [46] annealed self-ion irradiated W using both ex-situ and in-situ TEM annealing. For samples annealed ex-situ, the authors noted the loop size increased to $\sim 30 \mathrm{~nm}$ (see Figure 6) and loop density decreased to $\sim 10^{19}$ loops $/ \mathrm{m}^{3}$ (Figure 7) at an annealing temperature of $1000^{\circ} \mathrm{C}$ with no loops observed at $1400^{\circ} \mathrm{C}$. During the in-situ TEM annealing experiments (up to a temperature of $1200^{\circ} \mathrm{C}$ ), Ferroni et al. [46] reported an accelerated loss of loops which was attributed to the stage V recovery step and enhanced by the proximity of the free surfaces in the TEM foil. The authors [46] also reported the nucleation of voids at an annealing temperature of $800^{\circ} \mathrm{C}$, agreeing well with the neutron data presented in Figure 4.

\subsection{Light ion irradiation}

Figure 8 shows a summary of the damage microstructure of W irradiated in-situ with 15, 60 or 85 $\mathrm{keV} \mathrm{He}^{+}$to doses of 3.0 DPA at temperature of 500,750 and $1000^{\circ} \mathrm{C}$ reported by Harrison et al. [49]. At low temperature and low DPA the microstructure features are dominated by small loops (around $5 \mathrm{~nm}$ in diameter at 1.0 DPA, agreeing well with neutron data, see Figure 4) and He bubbles. Figure 9 and Figure 10 show the cavity diameters and volumetric densities reported under neutron $[32,33,35,36,41,47]$, self [46] and light ion [39,52-54] irradiations as a function of DPAs at a range of temperatures. This slightly larger dislocation size $(5 \mathrm{~nm})$ in Ref. [49] compared to previous neutron and self-ion irradiations (Figure 6) may be due to increased He bubble size and density (Figure 9 and Figure 10 ) removing vacancies from the flux leaving an excess of SIA clusters. As the irradiation temperature increases to $1000^{\circ} \mathrm{C}$ this shifts to a microstructure dominated by $\mathrm{He}$ bubbles with no dislocation loops, agreeing well with the neutron irradiation works on $\mathrm{W}$ [30-35]. At $1000^{\circ} \mathrm{C}$, above the Stage V recovery stage, interstitials and interstitial clusters will be highly mobile to sinks and vacancy clusters unstable to thermal emission of vacancies leading to the 
increased nucleation and growth of bubbles in this regime. However, the complete lack of dislocation loops observed at this temperature may also be enhanced by the proximity of the TEM foil surfaces providing a large concentration of sinks for rapidly mobile defects and defect clusters at this temperature.

The authors noted the presence of dislocation loops with $\boldsymbol{b}= \pm 1 / 2\langle 111\rangle$ and none with $\boldsymbol{b}=\langle 100\rangle$ contrary to self-ion irradiation work $[20,44,45]$ which will be discussed further in $\S 4.4$. In addition, the authors [49] reported a slight dominance for interstitial type loops $(\sim 60 \%)$ as compared with vacancy type $(\sim 40 \%)$ for irradiation temperatures of $500^{\circ} \mathrm{C}$ and the interstitial type loop percentage increased to $80 \%$ at the higher irradiation temperatures of $750^{\circ} \mathrm{C}$ which was attributed to more vacancies being trapped in He bubbles at the higher temperature and in good agreement with the insitu and near surface self-ion irradiations by Yi et al. [20,44,45]. Interstitial type loop population was also observed to increase with increases He appm, again due to higher number densities of bubbles removing vacancies form the flux. At an irradiation temperature of $500^{\circ} \mathrm{C}$ the authors [49] also noted the formation of He bubble lattices, similar to the void lattices found in neutron irradiated work and this will be discussed further in $\S 4.5$.

The formation of vacancy type-loops in the He ion irradiation case [49] is an intriguing one, opposing their nucleation mechanism from cascade collapse as in the $\mathrm{Au}[25]$ and $\mathrm{W}$ ion $[20,44,45]$ irradiations. Light ion irradiation $(\mathrm{H}$ or $\mathrm{He}$ ) will produce dilute cascades, mainly forming many isolated FPs [5]. As the irradiation temperature in the He ion irradiation case [49] were all at or above $500^{\circ} \mathrm{C}$ this will be above the Stage III recovery mechanism in W where monovacancies are mobile [46,51]. Thus, a mechanism whereby the vacancies accumulate and coalesce into extended defect clusters and dislocation loops via thermal migration can be envisaged. 


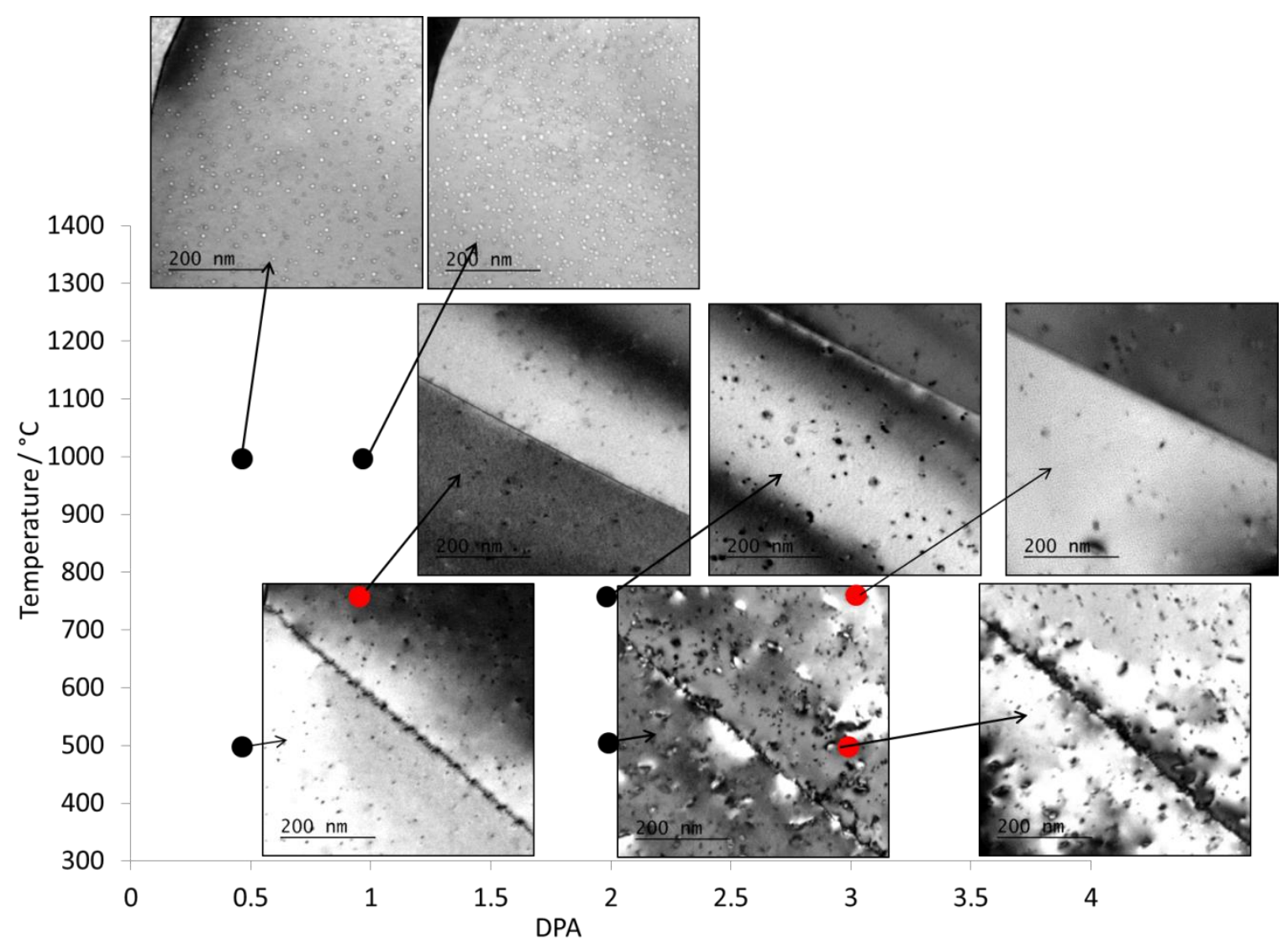

Figure 8. Summary of micrographs from He ion irradiated $W$ represented a function of the irradiation temperature and DPA reproduced from Ref. [49]

The response of $\mathrm{W}$ and $\mathrm{W}-5 \mathrm{Ta}$ alloys under both $3 \mathrm{MeV}$ and $40 \mathrm{keV} \mathrm{H}$ ion irradiation using ex-situ and in-situ irradiations respectively, at $350^{\circ} \mathrm{C}$ to a dose of $0.7 \mathrm{DPA}$ has been studied by Ipatova et al. [48]. Dislocation loop density $\left(\sim 8 \times 10^{21}\right.$ loops $\left./ \mathrm{m}^{3}\right)$ and size $(\sim 6 \mathrm{~nm})$ in the in-situ experiments [48] agreed well with work neutron irradiations by He et al. [47] at low doses 0.1 DPA (see Figure 6 and Figure 7). However, loop density decreased significantly to around $2 \times 10^{21} 100 p s / \mathrm{m}^{3}$ at 0.7 DPA under $40 \mathrm{keV} \mathrm{H}$ irradiation. The dramatic decrease in the density of dislocation loops in Ref. [48] compared to the neutron irradiations reported by Fukuda et al. [32] (as seen in Figure 7) may be attributed to the loops coalescing to larger diameters of $\sim 30 \mathrm{~nm}$ at 0.7 DPA compared to $\sim 3 \mathrm{~nm}$ (Figure 6) at 1.0 DPA for neutron irradiations [32]. The results from the ex-situ irradiations also showed the formation of dislocation tangles which were not observed in the in-situ case. This may be attributed to the accelerated loss of defect clusters and dislocation loops to the TEM foils free 
surfaces, however, in addition to this the damage rates between the in-situ and ex-situ irradiations varied over two orders of magnitude, $\left(10^{-4}\right.$ and $10^{-6} \mathrm{DPA} / \mathrm{s}$ respectively) and so the longer irradiation times required in the ex-situ case would give rise to dynamic annealing of defects and increased coalescence of dislocation loops to form entangled structures.

Dislocation loop analysis of the in-situ irradiated samples revealed that the dislocation loops were all of $\boldsymbol{b}=1 / 2<111>$ type, similar to the observations in Ref. [49]. In addition, only interstitial type dislocation loops were formed. This may be attributed to the irradiation temperature being lower than the Stage III recovery mechanism for monovacancy migration in $\mathrm{W}$ and thus vacancy-type loop nucleation by coalescence of thermally migrating vacancies cannot occur. The formation of a cavity lattice was observed W5Ta alloys irradiated with $3 \mathrm{MeV} \mathrm{H}$ at $350^{\circ} \mathrm{C}$ [52], with similar cavity sizes reported in neutron irradiations [35]. This also agreed well with He ion irradiations [49] but was unlike self-ion work of Yi. et al $[20,44,45]$ at similar temperatures (discussed further in §4.5.1). In further work, Ipatova et al. [39] post irradiation annealing the W-5Ta alloy using in-situ TEM and observed the formation of $\mathbf{b}=1 / 2<111>$ vacancy type loops at $\sim 600^{\circ} \mathrm{C}$. The higher temperature for vacancy type loop observation compared to the in-situ He ion irradiations in pure W [49] may be attributed to Ta hindering vacancy mobility through a vacancy-solute trapping mechanism [39]. 


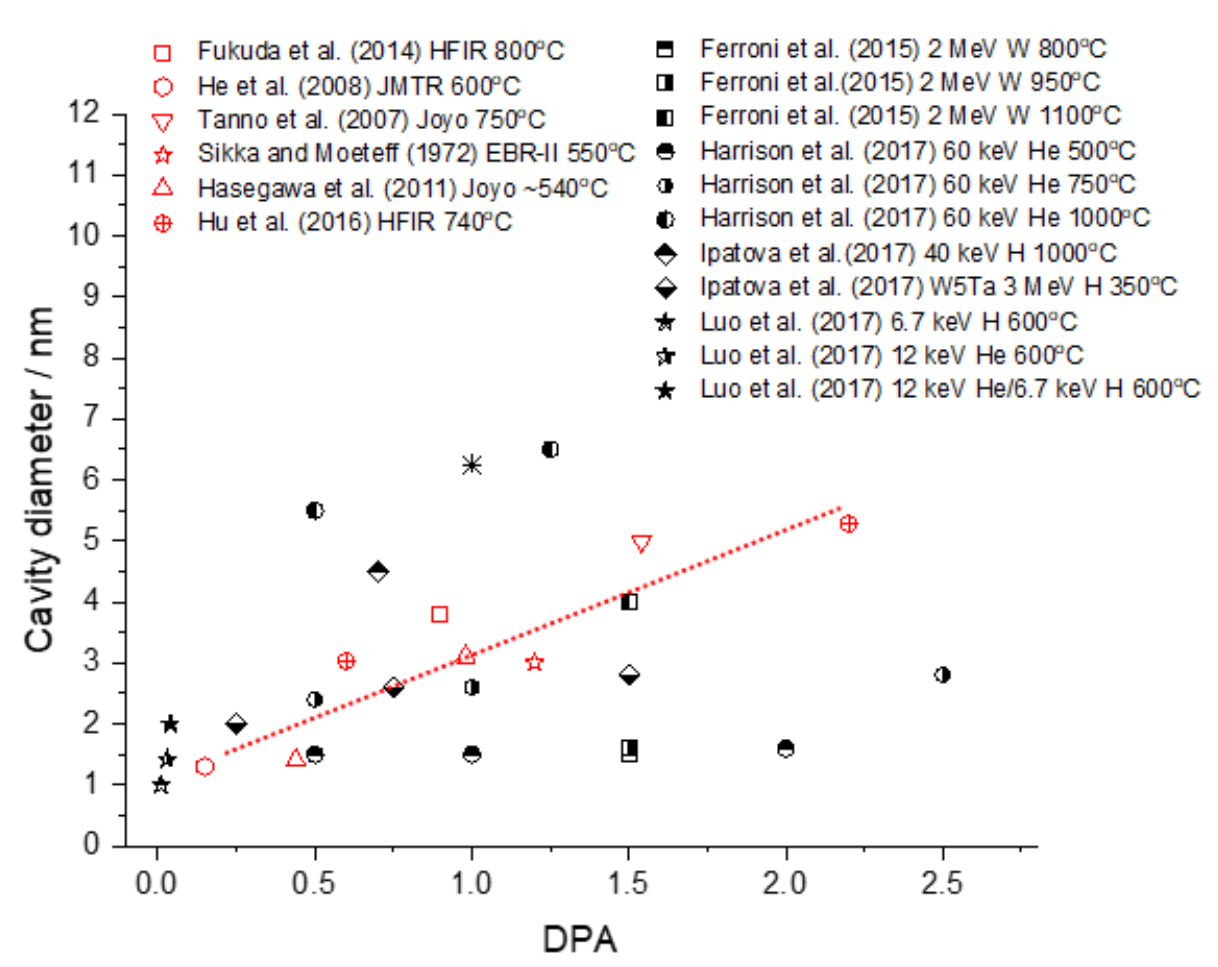

Figure 9. Plot of cavity (void/gas bubble) diameters for neutron irradiated W (open red symbols, line to guide eyes for neutron data only) $[32,33,35,36,41,47]$ and ion irradiated $W$ samples (semi/closed black symbols) $[39,46,52-54]$.

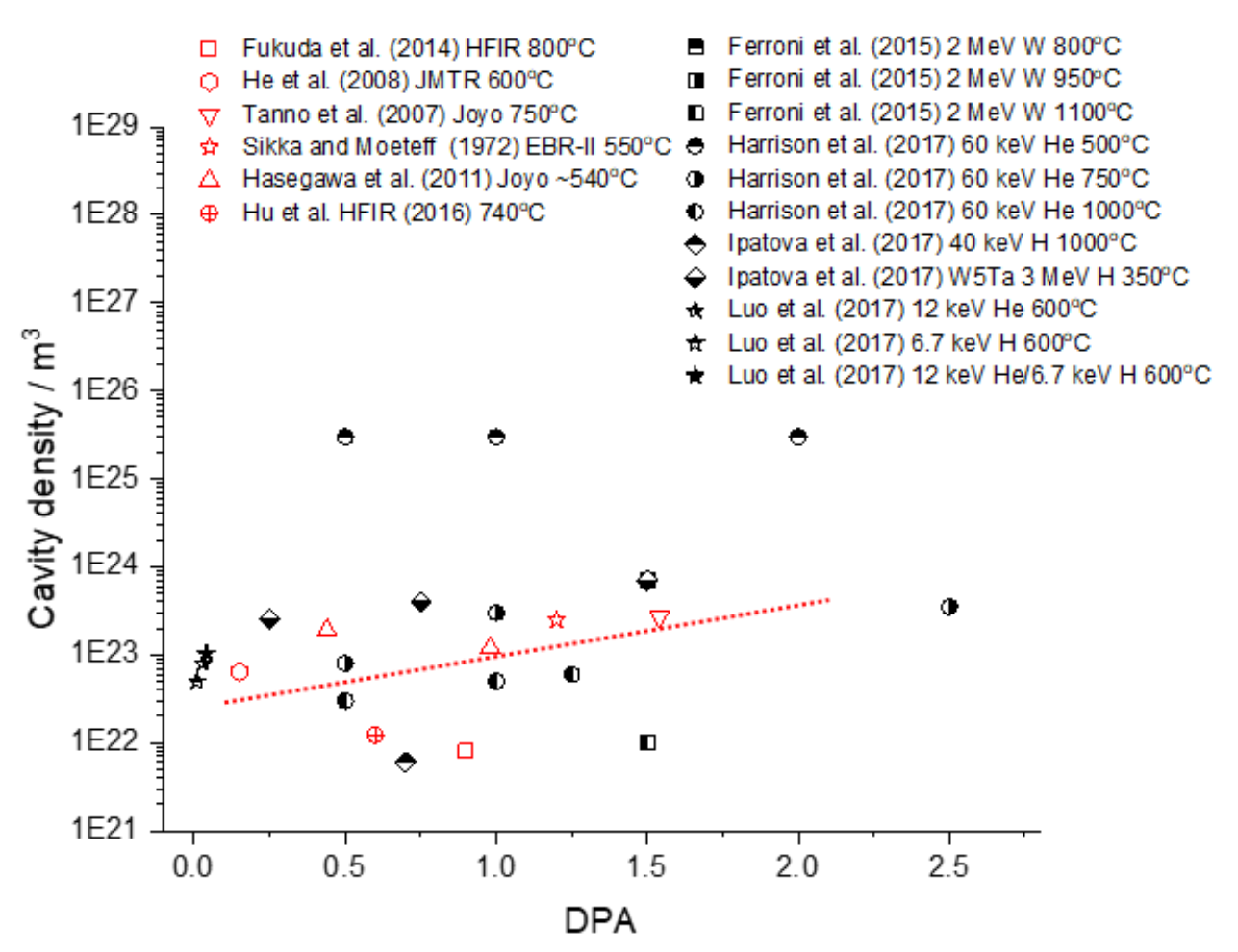

Figure 10. Plot of cavity (void/gas bubble) densities for neutron irradiated W (open red symbols, line to guide eyes for neutron data only) $[32,33,35,36,41,47]$ and ion irradiated $W$ samples (semi/closed black symbols) $[39,46,52-54]$. 
The effect of co-implantation of $\mathrm{H}$ with $\mathrm{He}$ on the nucleation of cavities has been investigated by Luo et al. [53]. Samples irradiated with $6.7 \mathrm{keV} \mathrm{H}$ ions (to a dose of $1 \times 10^{21} \mathrm{H} / \mathrm{m}^{2}$ ) at $600^{\circ} \mathrm{C}$ had an average bubble diameter of $\sim 1.0 \mathrm{~nm}$ (see Figure 9) and volumetric density of $\sim 5 \times 10^{22}$ bubbles $/ \mathrm{m}^{3}$ (see Figure 10). Samples irradiated with $12 \mathrm{keV}$ He ions (to achieve the same implantation depths at the $\mathrm{H}$ ions) to a dose of $5 \times 10^{19} \mathrm{He} / \mathrm{m}^{2}$ at $600^{\circ} \mathrm{C}$ had a larger average bubble diameter $(\sim 1.4 \mathrm{~nm})$ and higher volumetric density $\left(\sim 8 \times 10^{22}\right.$ bubbles $\left./ \mathrm{m}^{3}\right)$ than the $\mathrm{H}$ ion irradiations, likely due to the lower solubility of $\mathrm{He}$. Sequential $\mathrm{He} / \mathrm{H}$ irradiations (to a dose of $5 \times 10^{19} \mathrm{He} / \mathrm{m}^{2}$ and $1 \times 10^{21} \mathrm{H} / \mathrm{m}^{2}$ ) led to an average bubble diameter of $\sim 2.0 \mathrm{~nm}$ and volumetric density of $\sim 1.0 \times 10^{23}$ bubbles $/ \mathrm{m}^{3}$ (see comparisons in Figure 9 and Figure 10). The authors attributed this to increased trapping of $\mathrm{H}$ by pre-existing He bubbles leading to greater retention of $\mathrm{H}$ in the sample.

These results show there are important synergistic effects of $\mathrm{H}$ and $\mathrm{He}$ on cavity nucleation which will both be produced from transmutation reactions in the bulk of the divertor structure, as well as implantation from the plasma at the divertor surface. However, the bubble sizes were much smaller and, densities much larger than reported for neutron irradiations at similar temperatures [30-35] (see Figure 4). This is likely due to the large concentration of implanted $\mathrm{He}$ and $\mathrm{H}$ creating a high density of bubble nucleation sites. With regards to the lack of voids observed in self-ion irradiations $[20,45,55]$, a combination of sequential or simultaneous $\mathrm{H} / \mathrm{He}$ ions to concentrations anticipated from transmutation reactions under fusion relevant conditions may aid better reproduction of void formation in neutron irradiated $\mathrm{W}$.

\subsection{Dislocation loop nucleation under different projectiles}

It has been reported that the formation of energetically less favourable $\boldsymbol{b}=<001>$ dislocation loops occurs almost exclusively in Fe alloys [19], whereas irradiated microstructures of W [56] and Mo [43] are dominated by the energetically more favourable $\boldsymbol{b}= \pm 1 / 2<111>$ dislocation loops. From the discussion in $\S 3$ it would also be expected that the microstructure would contain a majority of $\boldsymbol{b}=$ $\pm 1 / 2<111>$ dislocation loops, unless $<100>$ type loops form via Eq. 3 . 
Thus, the observation of $\boldsymbol{b}=\langle 100\rangle$ loops by Yi et al. [44,45] in self-ion irradiated W and the lack of those loops in $\mathrm{He}, \mathrm{H}$ and $\mathrm{D}$ ion irradiations at the same temperature $[48,49,56,57]$ may be attributed to two mechanisms;

- The lack of dense cascade damage in the light ion irradiation case, as compared with selfions, reducing the energy density deposited into the material and thus the magnitude and lifetime of the thermal spike.

- The presence of gas atom impurities pinning mobile $\boldsymbol{b}= \pm 1 / 2<111>$ dislocation loops from reacting as observed in $\mathrm{BCC}$ Fe alloys $[21,58]$.

Pre-implantation of He has been showing to have no effect on the Burgers vector of the dislocation loops formed in $\mathrm{W}$ under self-ion irradiation at $500^{\circ} \mathrm{C}$ by Harrison et al. [59]. Pristine $\mathrm{W}$ and samples pre-implanted with 1500 appm He were irradiated using $350 \mathrm{keV} \mathrm{W}$ ions to a dose of 3.0 DPA at $500^{\circ} \mathrm{C}$. Dislocation loops with $\boldsymbol{b}= \pm 1 / 2<111>$ and $\boldsymbol{b}=\langle 100>$ were observed in both the pristine $\mathrm{W}$ samples and those pre-implanted with He with similar percentages of $\boldsymbol{b}=\langle 100\rangle$ loops in both cases. This is in contrast to the work performed using only He ion irradiation where no $\boldsymbol{b}=\langle 100\rangle$ type loops were observed. Under these aforementioned irradiation conditions using $\mathrm{He}$ [49,57], H [52] and D [56] ions only, there will be dilute cascades formed with many isolated FPs [5]. Thus, the lack of $\boldsymbol{b}=\langle 100\rangle$ type loops in previous light ion irradiations, He [49,57], H [52] and D [56] was not due to the presence of impurity gas atoms inhibiting migration and reaction of $\boldsymbol{b}= \pm 1 / 2<111>$ loops (as shown in $\mathrm{FeCr}$ alloys [21]) but rather to the absence of dense cascade damage, which is in good agreement with predictions from MD simulations [27].

Thus, there are also significant consequences when considering PKA energy and resulting cascade energy densities, when aiming to replicate neutron damage mechanisms in $\mathrm{W}$ using ion beam(s). The increase in the percentage of $\boldsymbol{b}=\langle 100\rangle$ type loops as a function of cascade density could also have large implications for the radiation behaviour and mechanical degradation of materials. As previously discussed, dislocation loops with $\boldsymbol{b}= \pm 1 / 2<111\rangle$ are glissile and those with $\boldsymbol{b}=\langle 100\rangle$ are sessile for BCC materials [19-21] and so the former may more readily migrate to sinks or, annihilate 
with other defects. However, the latter, being immobile will accumulate in the microstructure, further degrading the materials mechanical properties and the populations of loop types must be captured accurately by ion beam irradiations as to emulate mechanical degradation of neutron irradiated materials. Further to this, in the sequential He+W ion irradiation from Ref. [59] no He bubble nucleation was observed. This is contrary to single He ion beam irradiation to the same dose and temperature $\left(3.0 \mathrm{DPA}, 500^{\circ} \mathrm{C}\right)$ which produced a He bubble lattice [60] and neutron irradiations under similar temperatures and doses, where void lattices form [33-35]. This indicates a simultaneous irradiation with $\mathrm{He}$ and $\mathrm{W}$ ions may more accurately replicate both aspects of dislocation loop type populations and cavity lattice formation.

\subsection{Cavity lattice formation}

\subsubsection{Formation mechanism}

The mechanism of how cavity lattices nucleate under irradiation has received many studies in the literature $[5,33-35,50,61-71]$ with models being based on elastic interaction between gas bubbles, interstitial loop punching or anisotropic 1-D SIA diffusion and 2-D SIA diffusion. In the case of anisotropic SIA migration mechanisms, during cavity nucleation, those cavities which are aligned experience a shadowing effect from the anisotropically migrating SIAs resulting in a lattice. In the BCC unit cell, 1-D SIA diffusion involves the migration of a $<111>$ dumbbell or crowdion along the close packed direction (shown in Figure 11a). However, void lattices in neutron irradiated Mo do not show alignment along <111> directions, contesting this theory [72]. A 2-D SIA diffusion mechanisms was proposed by Evans [69,71] whereby the diffusing SIA cluster is a $<110>$ type dumbbell which migrates within $\{110\}$ planes (shown in Figure 11b) which is consistent with the strong ordering of cavity lattices on $\{110\}$ planes in irradiated BCC metals. These $<110\rangle$ type dumbbell SIA configurations are forbidden from rotating onto another $\langle 110\rangle$ axis which would result in 3-D diffusion, as shown in Figure 11c. However, this is inconsistent with recent density functional theory (DFT) simulations, where the <111> crowdion was reported to be the lowest 
energy SIA for non-magnetic BCC metals [73]. Thus, the mechanism of formation is still under much debate.

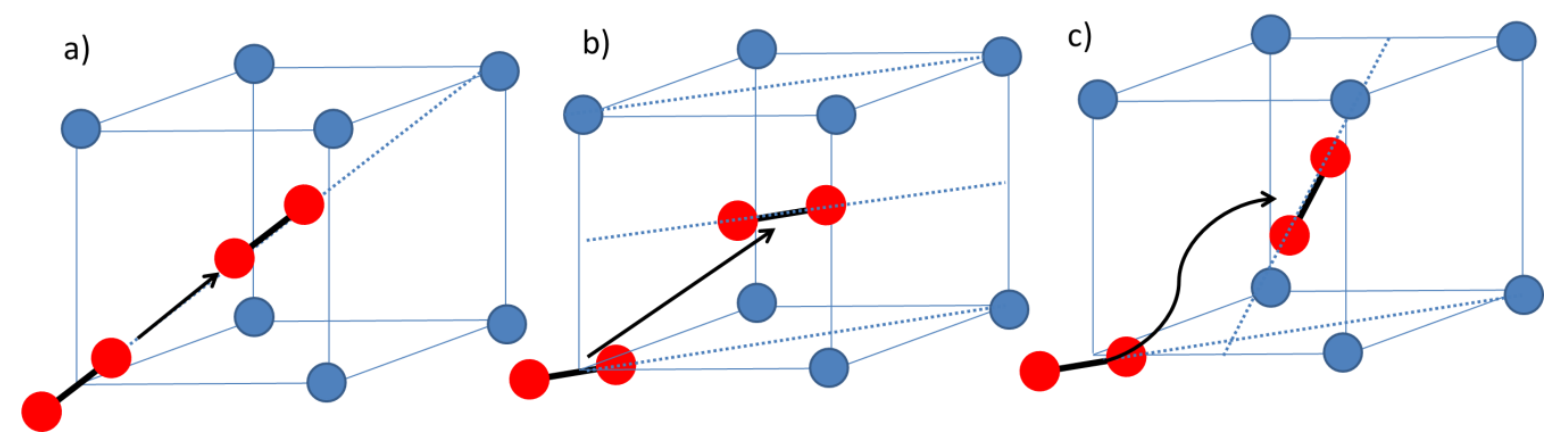

Figure 11. a) Diffusion of $<111>$ dumbbell along $<111>$ direction in the BCC unit cell (1-D SIA model), b) diffusion of $\langle 110\rangle$ dumbbell along the (110) plane (2-D SIA diffusion model), c) forbidden rotation of $\langle 110\rangle$ dumbbell onto (011) plane (figure reproduced from Ref. [60])

In either case, the mechanism of cavity lattice formation must be active in both the neutron works described in $\S 4.1$ and the $\mathrm{H} / \mathrm{He}$ ion irradiations in $\S 4.3$. It is then, however, intriguing as to why these structures were not formed in the self-ion irradiations described in $§ 4.2$. Partially ordered void lattices have been observed in self-ion irradiated Al pre-implanted with He [74], showing that injected interstitials should have no effect on the formation of these lattices. As noted previously the presence of gas impurity atoms has been observed to aid the formation and alignment of these lattices [50] which will be lacking in the work by Yi et al $[44,45]$.

\subsubsection{Effect of helium gas}

Voids of around $3 \mathrm{~nm}$ in diameter with a lattice spacing of $19.5 \mathrm{~nm}$ were reported by Sikka and Moteff [35] in neutron irradiated W to a dose of $\sim 10^{22} \mathrm{n} . \mathrm{cm}^{-2,}$ at $550^{\circ} \mathrm{C}$. A void lattice was also found in $\mathrm{W}$ irradiated in the Joyo reactor at $750^{\circ} \mathrm{C}$ to a similar fluence by Tanno et al.[33,34] who reported cavity lattice parameters with a void diameter of $5 \mathrm{~nm}$ and void lattice spacing of $20 \mathrm{~nm}$. The increased irradiation temperature may have resulted in the slightly larger void diameter found by Tanno et al.[33,34] when compared to the results of Sikka and Moteff [35].

Helium bubble lattices have been reported to form in W irradiated ex-situ with $50 \mathrm{keV}$ He ions [66]. The helium bubble diameter was $2 \mathrm{~nm}$ and the bubble lattice constant of $6.2 \pm 0.2 \mathrm{~nm}$ was determined 
from selected area diffraction patterns (SADPs) and image analysis. Harrison et al. [60] have also reported the formation of He bubble lattices in W formed under in-situ irradiation with 15, 60 and $85 \mathrm{keV} \mathrm{He}$ ions at $500^{\circ} \mathrm{C}$, reporting an increase in the bubble lattice spacing from $\sim 4.6$ to $8 \mathrm{~nm}$ with increasing ion energy. Figure 12a and b shows a void lattice reproduced from Ref. [35] under neutron irradiation and $\mathrm{He}$ bubble lattice formed under $15 \mathrm{keV} \mathrm{He}$ ion irradiation to 3.0 DPA from Ref. [60], showing the difference in cavity lattice spacing and cavity size between the two irradiation conditions, which has been observed in other BCC metals [33-35,50,57,66].
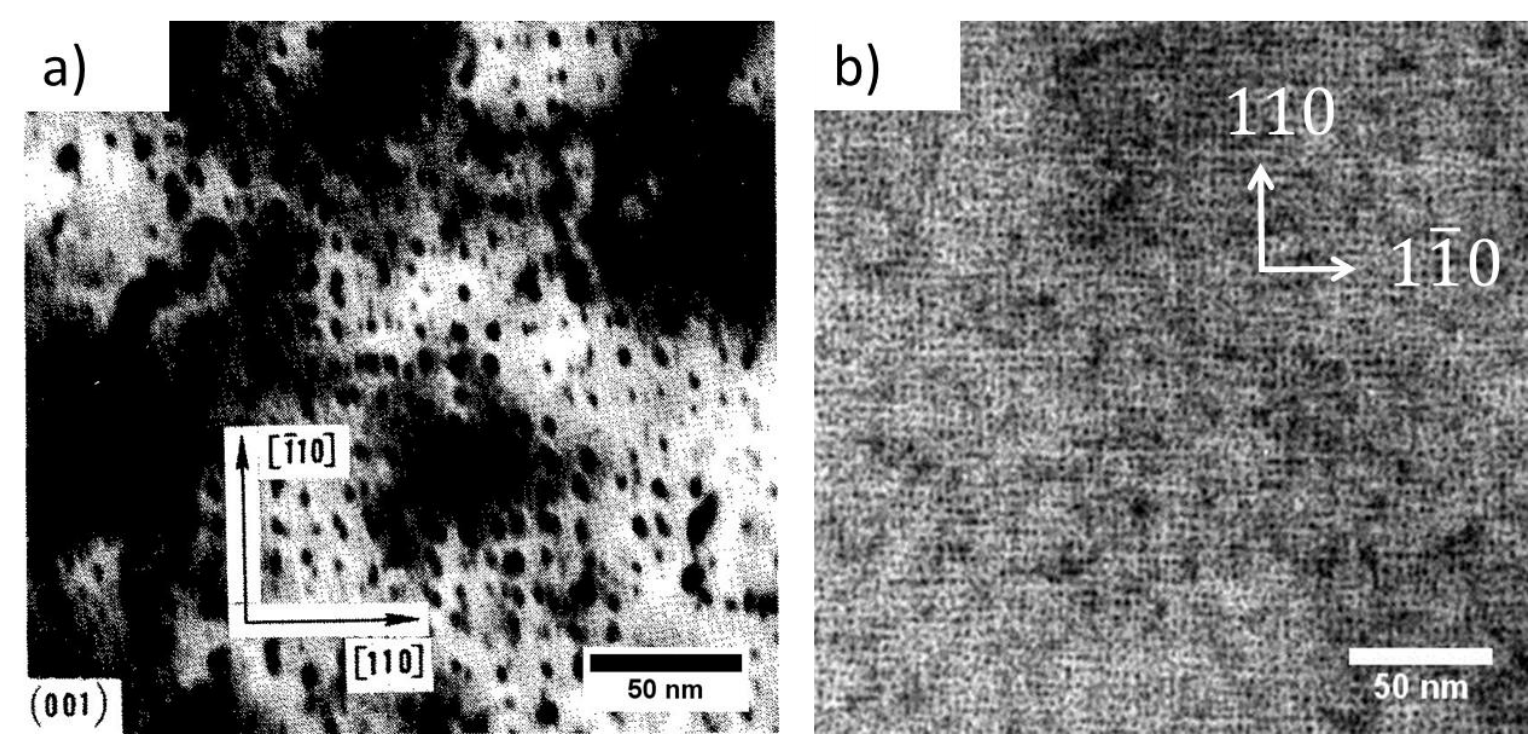

Figure 12. a) A void lattice in W irradiated with neutrons to a dose of $\sim 1.5$ DPA $\left(\sim 10^{22} \mathbf{n . c m}^{2}\right)$ at $550{ }^{\circ} \mathrm{C}$ showing ordering along $\{110\}$ planes observed from Ref. [35] and b) a He bubble lattice in W irradiated with $15 \mathrm{keV}$ He ions to a dose of 3.0 DPA $\left(1.1 \times 10^{17}\right.$ ions.cm $\left.^{2}\right)$ from Ref. [60].

The spacing of the He bubble lattice was found to increase with increasing $\mathrm{He}^{+}$ion energy in in-situ TEM irradiations (i.e. decreasing amount of He implantation and concentration), from $\sim 4.3 \pm 0.5 \mathrm{~nm}$ to $9.3 \pm 0.9 \mathrm{~nm}$ for 15 and $85 \mathrm{keV} \mathrm{He}$ respectively in work by Harrison et al. [60]. The decrease in lattice parameter with increasing He content was attributed to He inhibiting mobility of vacancies, resulting in a higher density of smaller bubbles in the random, disordered phase before ordering occurs from diffusing SIAs. As the He concentration is reduced the mean free path of vacancies is increased due to the lower probability of pinning leading to a less dense array of random bubbles which, when ordered, forms a bubble lattice with a larger lattice spacing. 
With the previous sections discussing the nucleation mechanisms and the types of defect formed under different irradiating projectiles and conditions, the effects these have on the materials mechanical and thermal properties will now be discussed.

\section{Property changes under irradiation}

\subsection{Hardness}

\subsubsection{Neutron irradiation hardening}

Hardness increase of neutron irradiated $\mathrm{W}$ as a function of dose under different neutron spectra and temperatures are shown in Figure $13[17,33,36,41,47]$ (open symbols indicate thermal neutron spectra and half-filled symbols represent fast neutron spectra) showing good agreement in the literature. Hu et al. [41] examined the change in Vickers hardness $\left(\Delta \mathrm{H}_{\mathrm{v}}\right)$ of W irradiated in HFIR at temperatures from $90-850^{\circ} \mathrm{C}$ to doses of between 0.03-2.2 DPA. The authors [41] found that although there was an increasing trend for hardness with irradiation dose, there was minimal effect of temperature on the doses studied. The authors attributed the increase in hardness at low DPA $(<0.3$ DPA) to the nucleation and saturation of voids and dislocation loops. The increases in hardness thereafter $(>0.6 \mathrm{DPA})$ were attributed to the formation of intermetallic W-Re-Os precipitates. However, it is important to note that the authors exclusively attributed the contribution of $\boldsymbol{b}=$ $\pm 1 / 2<111>$ dislocation loops in their analysis of $\Delta \mathrm{H}_{\mathrm{v}}$ no detailed dislocation loop analysis is reported from their TEM analysis.

Comparison of hardness increases from neutron irradiated $\mathrm{W}$ to doses of $0.15 \mathrm{DPA}$ at $600^{\circ} \mathrm{C}$ in the JMTR and proton irradiated $\mathrm{W}$ samples to the same DPA at $500^{\circ} \mathrm{C}$ using $1 \mathrm{MeV} \mathrm{H}$ ions has been performed by He et al. [47]. The authors reported that the increase in $\Delta \mathrm{H}_{\mathrm{v}}$ for the neutron and proton irradiated W samples were 2.0 and $1.8 \mathrm{GPa}$ respectively. Hasegawa et al. [36] compared the hardness increase of W and W-Re samples irradiated in HFIR, JMTR and Joyo as to elude to the effect of neutron spectra on the change in mechanical properties. However, the authors report sporadic data points and the respective increase in the hardening of pure $\mathrm{W}$ under different neutron spectra at 


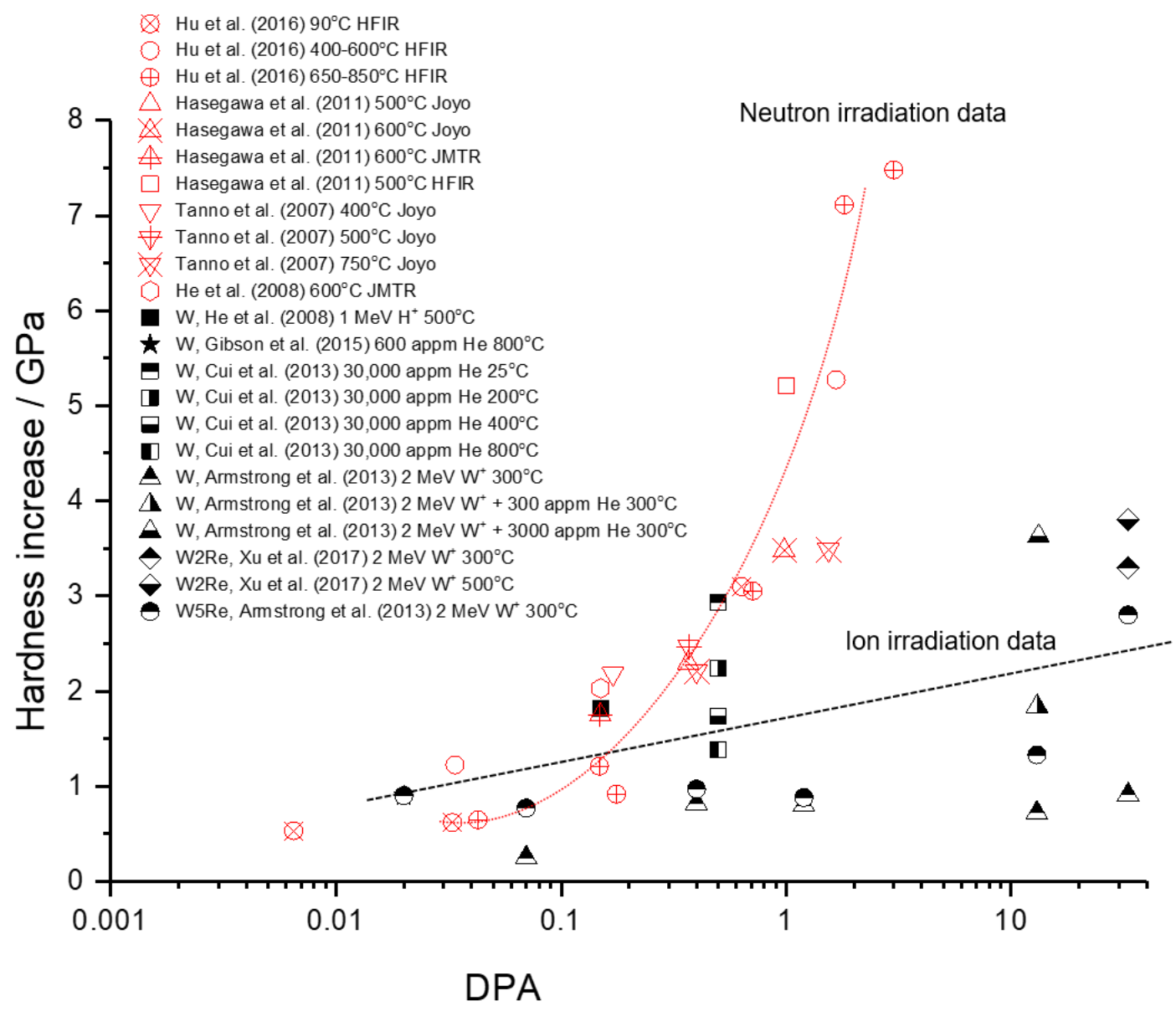

Figure 13. Hardness increase as a function of dose for neutron irradiated $\mathbf{W}$ samples under different conditions $[33,36,41,47]$ (open red symbols) and for ion irradiated $W$ and $W$ alloy samples under different conditions $[47,79-83]$ (closed/semi-filled black symbols). Lines to guide eyes only.

different temperatures. This makes comparison difficult as it doesn't elude to any trend. However, they discussed the saturation of the irradiation hardening of pure $\mathrm{W}$ due to the formation of void lattices which have been reported to stabilise the irradiation damage structure [50]. The authors [36] report that the increase in hardening in $\Delta \mathrm{H}_{\mathrm{v}}$ for the pure W in HFIR at 1.0 DPA is and $~ 5.2 \mathrm{GPa}$ in Joyo is $\sim 3.4 \mathrm{GPa}$ which the authors attribute to the increased radiation hardening in HFIR from the higher absorption cross section of $\mathrm{W}$ for thermal neutrons leading to increased transmutation precipitation. Tanno et al. [33] examined the effect of transmutation elements on the radiation hardening of $\mathrm{W}$ and $\mathrm{W}$ alloys under neutron irradiation. The authors irradiated samples in the Joyo reactor with fast neutrons $(>0.1 \mathrm{MeV})$ at temperatures between $400-750^{\circ} \mathrm{C}$ to doses of $1.54 \mathrm{DPA}$. The lower increase in hardening from Tanno et al. [33] for the sample irradiated to 1.54 DPA at 
$750^{\circ} \mathrm{C}$ compared to the other data in Figure 13 may again be due to the lower transmutation rate of $\mathrm{W}$ in a fast spectrum leading to less second phase precipitation hardening.

Since the effect of fusion neutrons remains experimentally unavailable there has been a concerted effort to simulate the complex effects of radiation damage and transmutation in the modelling community [75-78]. Marian et al. [76] discussed the need for a multiscale approach when aiming to reproduce the evolution of the many kinds of irradiation defects and transmutation species in $\mathrm{W}$ using computer simulation to accurately capture their interaction and synergism. Huang et al. [75] simulated the radiation hardening of $\mathrm{W}$ due to Re precipitation with a combination of MD simulations, neutron transport codes and stochastic cluster dynamics. The authors [75] compared against experimental results from irradiations in the Joyo fast reactor and HFIR as well as extrapolating their work to conditions anticipated in DEMO under temperatures of $400-800^{\circ} \mathrm{C}$ to 2.0 DPA. Under DEMO conditions, Huang et al. [75] predict hardness increases of over $5 \mathrm{GPa}$ for all temperatures at 2.0 DPA. The authors [75] found that in all cases the increase in hardening was always dominated by Re precipitates, and although hardness increases under HFIR matched well with experimental results from $\mathrm{Hu}$ et al. [41] the dominance of hardening from Re was contrary to experimental observations in Ref. [41], which reported significant increase in hardening at this dose due to void density. This failing in the modelling prediction was attributed to insufficient understanding of the underlying physics of defect-solute interactions in the W-Re system [75]. Thus, as hardening mechanisms are dominated by both Re precipitation and from void density, both need to be correctly emulated in ion beam irradiations to accurately capture hardening mechanisms in neutron irradiations.

One puzzling experimental observation described in $\S 4.1$ is the precipitation of $\sigma$ and $\chi$ phases at concentrations and temperatures lower than the solubility limit $[32,76]$. First principles DFT simulations of W-solute interstitial defects in W alloys has been performed by Gharaee et al. [78] to examine the effect of radiation induced defects on sub-solubility limit precipitates. The authors [78] showed the mixed W-Re interstitial adopts the $<111>$ SIA configuration and is strongly attracted to 
each other with a binding energy of $-3.2 \mathrm{eV}$. However, the mixed interstitial has a large defect formation volume of $\sim 1.6$ times the volume per atom of the ideal structure which is associated with a strain field along the $<111>$ direction. This induced strain field gives rise to alignment of other mixed interstitials along parallel first-neighbour $<111>$ strings and is proposed to act as the precursor for the formation of the needle like intermetallic precipitates formed in neutron irradiations. In the experimental reports of precipitate alignment along $\{110\}$ planes observed by TEM, the samples were imaged along the $<001>$ zone axis $[33,34]$. As there is coincidence between the $<111>$ type directions and $\{110\}$ type planes in this orientation in BCC this observation is consistent with precipitate growth along $<111>$ directions from [78].

Huang et al. [77] used kMC and DFT simulations to study the nucleation and growth of Re clusters in W-2Re alloys. The authors [77] report that mixed interstitial diffusion is dominant in providing the agglomeration of $\mathrm{W}-\mathrm{Re}$ clusters which migrate via random $<100>$ hops in $3-\mathrm{D}$, unlike the SIA which migrates in fast sequences of mainly $1-\mathrm{D}<111>$ transitions. These clusters coalesce further with mixed interstitials, bringing more solute atoms and, subsequently, vacancies which recombine at the precipitate and increase solute concentration over time. However, as the arrival of further mixed interstitials delivers $\mathrm{W}$ atoms to the precipitate the maximum concentrations reported in Ref. [77] of Re were 50 at.\% near the core of the precipitate. This mechanism provides the concentration of Re required for precipitation of $\sigma$ phase (46 at.\% Re), however, it does not account for the formation of $\chi$ phase ( 75 at.\% Re) showing there is still further mechanistic understanding needed to explain the formation of these concentrated Re phases under neutron irradiation.

\subsubsection{Ion irradiation hardening}

Due to the nature of the damage profile of ion irradiation experiments, conventional Vickers hardness measurements are not possible as penetration into the pristine material under the ion irradiated layer occurs. Thus, nanoindentation measurements are used giving a range of hardness increase measurements as a function of indenter displacement depth, making comparison difficult due to effects arising from the size of the plastic zone. Figure 13 shows there is a large degree of scatter in 
hardness increase measurements of W and WRe alloys under different ion irradiation conditions (irradiation projectile, temperature and dose) [47,79-83] and no study has, as yet, reproduced the hardness increases from neutron experiments (Figure 13) even to the high doses studied (10's DPA) in the ion irradiation case.

However, the large degree of scatter may arise from indentation size effects (ISEs) and the applicability of nanoindentation studies of shallow ion irradiated layers due to the non-uniform dose as a function of depth. Krumwiede et al. [84] performed Vickers and nanoindentation measurements on neutron irradiated steel alloys in the aim of spanning the two length scales to determine the applicability of how nanoindentaiton measurements of ion irradiation layers truly represented bulk properties. The authors [84] used a Nix and Gao model [85] to determine the ISE for each condition, finding that ISEs reduced after irradiation (where weaker size effects are observed with increasing defect density [85]) but were still prevalent at indentation depths of $0.8-1.0 \mu \mathrm{m}$. In Fe ion irradiated T91, $800 \mathrm{H}$ and Fe10Cr alloys, ISEs and non-uniform ion irradiated layer dose effects in nanoindentaiton measurements have been deconvoluted by Kareer et al. [86]. The authors used the Nix and Gao [85] model coupled to a radiation hardening term which was modelled as a simple power law function of DPA as a function of depth calculated from SRIM. A similar approach may also be applied to nanoindentation measurements of ion irradiated $\mathrm{W}$ in the aim of deconvolution of ISEs and extracting radiation hardening values as a function of depth (i.e. DPA) to reduce possible sources scatter.

The hardness increase of $2 \mathrm{MeV}$ self-ion irradiated $\mathrm{W}$ and $\mathrm{W}-5 \% \mathrm{Re}$ at irradiation temperatures of around $300^{\circ} \mathrm{C}$ up to doses of 33 DPA was studied by Armstrong et al. [79]. The authors [79] noted a saturation in the increase in hardness of pure $\mathrm{W}$ at a dose of 0.4 DPA which was correlated with TEM studies showing a saturation in dislocation loop density, consisting of a mixture of $\boldsymbol{b}= \pm 1 / 2<111\rangle$ and $\langle 100\rangle$ type loops. This agrees well with the work of $\mathrm{Hu}$ et al. [41] who attributed the increase in hardness at low DPA $(<0.3$ DPA) to the nucleation and saturation of voids and dislocation loops and further increases in hardness (>0.6 DPA) to the precipitation of intermetallic W-Re-Os 
precipitates. No void nucleation was reported in their work [79], even up to the high doses studied. The lack of void observation in self-ion work has been observed previously $[45,55]$ which may be attributed to the lack of gas impurity atoms to stabilise the void embryo (as compared to neutron irradiation experiments which will be produced from transmutation reactions) or, the injection of interstitial atoms supressing void nucleation and growth to visible sizes in the TEM. This difference in damage microstructure may have consequences when comparing hardening data from ion beam experiments employing self-ions and neutron irradiations.

Nanoindentation studies of sequentially irradiated W samples with $2 \mathrm{MeV}$ W ions to 13 DPA and then with He ions to a concentration either 300 or 3000 appm (increasing DPA by 0.02 and 0.25 DPA respectively) at a temperature of $300^{\circ} \mathrm{C}$ has been studied by Armstrong et al. [82]. The hardness increase was a factor of two higher for the high dose helium concentrations (3000 appm He). The authors attributed this to a combination of hardening mechanisms from dislocation loop and Hevacancy complexes. No He bubbles were observed in the work, however, and so the authors [82] surmised that all $\mathrm{He}$ would be trapped in pre-existing small vacancy clusters from the $\mathrm{W}$ ion irradiation.

Another effect of neutron irradiation not replicated in the self-ion irradiation of $\mathrm{W}$ is the formation of transmutation products such as Re, Ta and Os which precipitate as intermetallic phases and induce further hardening as a function of DPA which may explain the saturation of hardening in Ref. [79]. Neutron induced transmutation precipitation has been attempted to be physically modelled using ionirradiation by employing W-Re or W-Re-Os alloys as the starting materials and measuring their precipitation rate as a function of dose and thus the effect on the hardness $[20,32,79,81,87]$. Armstrong et al. [79] noted a large increase in the hardness ( 2.8 GPa) for the W-5\%Re samples irradiated under the same conditions as the pure $\mathrm{W}$ which was attributed to the precipitation of small (3-5 nm) Re clusters as determined from atom probe tomography (APT). However, the nucleation of these precipitates occurs at a much later irradiation dose in the self-ion irradiation as compared with neutrons $[33,36,41]$. Equally, the Re clusters formed under ion irradiation are generally very 
small dilute clusters that are at least semi-coherent with the matrix at very high DPAs (10's of DPA) [81]. The classical equiaxed $\sigma$ and needle like $\chi$ phase precipitation in neutron irradiations at lower doses (1.0-3.0 DPA), which are incoherent with the matrix and will lead to differing hardening mechanisms [30,33] compared with the ion-irradiation (small, coherent BCC Re clusters). The mechanism by which its proposed these precipitates will grow is by the arrival of $\mathrm{W}$-Re mixed interstitials migrating along $\langle 100\rangle$ hops in $3-\mathrm{D}$ and annihilation with vacancies at the precipitate, leading to an enrichment in $\operatorname{Re}[78]$. As the mixed W-Re interstitial adopts a <111> SIA configuration associated with a large strain field along the $\langle 111\rangle$ direction, it is suspected to lead to the nucleation of needle-like precipitates [78]. Thus, presence of large amounts of Re in the doped alloy in the ion beam case or, the high dose rates of ion beam experiments, may both serve to hinder this nucleation and growth mechanism of intermetallic precipitates in ion irradiated $\mathrm{W}$.

However, it has recently been reported that $\sigma$ and $\chi$ phases do form in W26Re alloys under $350 \mathrm{keV}$ $\mathrm{Ne}$ ion irradiations performed at 500 and $800^{\circ} \mathrm{C}$ by Harrison et al. [88]. Figure 14 shows a comparison of precipitate structure in neutron irradiated W at 1.0 DPA [32] with those formed at 5.0 DPA under Ne ion irradiation at 5.0 DPA at (both irradiations performed at $800^{\circ} \mathrm{C}$ ) showing similar sizes and lengths of the classical $\chi$-phase needle like structures. The authors [88] attributed to the higher DPA required to form precipitates of similar sizes to the shorter irradiation time in the ion beam case and as it was performed in-situ, the proximity of the free surfaces removing migrating WRe SIA clusters from the flux. The absence of $\sigma$ and $\chi$ phase formation under the previous high energy self-ion irradiations (discussed above) was attributed to the maximum PKA energy being an order of magnitude higher $(>2 \mathrm{MeV}$ in the self-ion irradiations compared with $400 \mathrm{keV}$ in a FBR neutron spectra). This will lead to a greater number of high energy density cascades and may promote ballistic mixing of migrating WRe SIAs back into the matrix, which has been observed to supress Cr precipitation in $\mathrm{FeCr}$ alloys $[89,90]$. Thus, the authors [Harrison] attributed the precipitation in the $350 \mathrm{keV} \mathrm{Ne}$ irradiation case to it better matching the PKA spectrum (maximum PKA transfer of 
around $120 \mathrm{keV}$ ) to neutron irradiations. However, further studies of the effect of projectile mass and energy, Re content and DPA on the rate of precipitation in WRe alloys in much warranted.

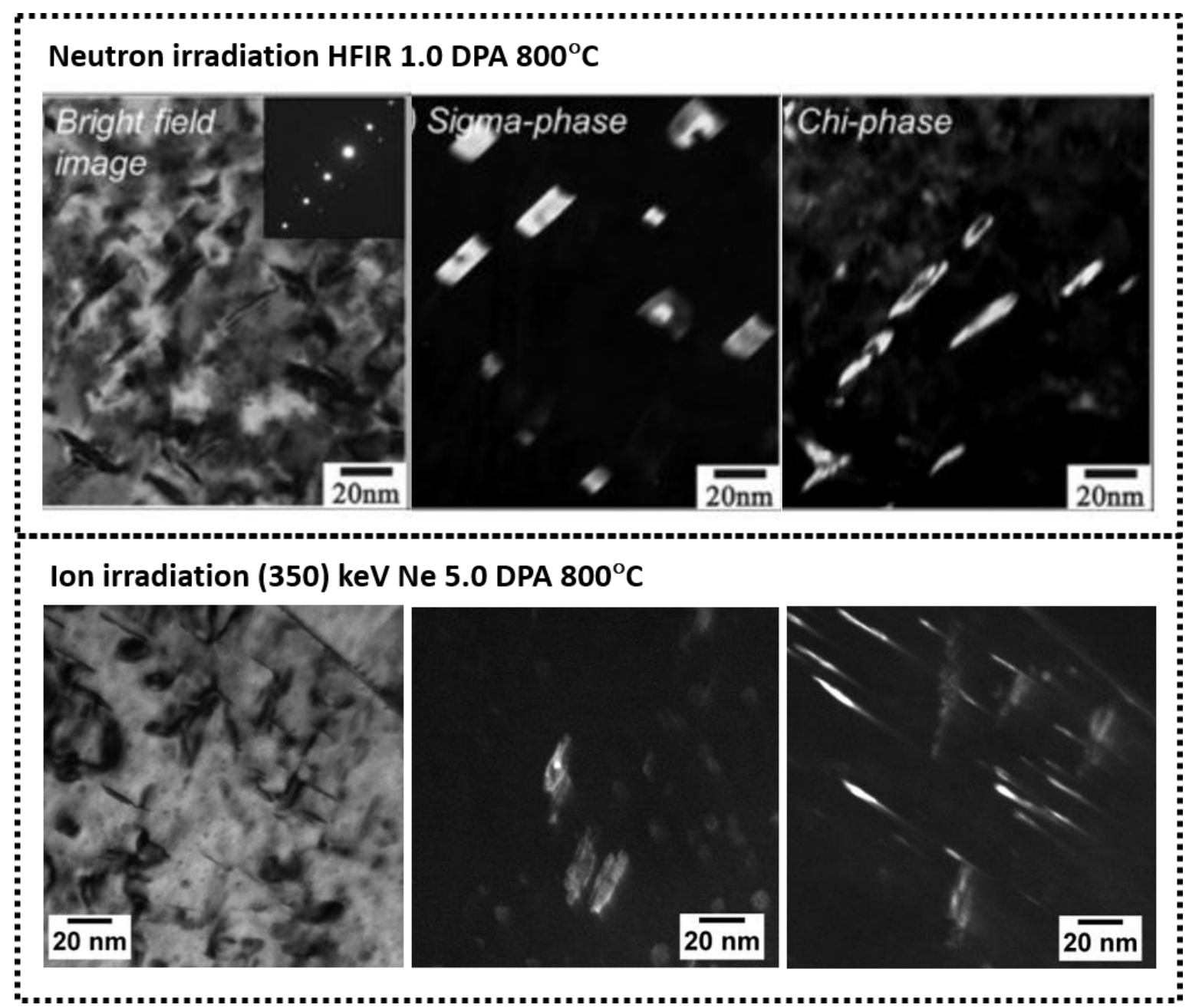

Figure 14. (Top row, left to right) BF-TEM image of pure $W$ irradiated in HFIR showing Re precipitate and dark-field (DF)-TEM images of $\sigma$-phase and $\chi$ phase precipitates [32]. (Bottom row, left to right) BF-TEM image of W26Re alloy irradiated to with $350 \mathrm{keV}$ Ne ions and DF-TEM images of $\sigma$ and $\chi$ phase precipitates [88].

The effect of $2 \mathrm{MeV} \mathrm{W}$ ion irradiation at 300 and $500^{\circ} \mathrm{C}$ to doses of $33 \mathrm{DPA}$ on the irradiation and precipitation hardening of W-2Re, W-4.5Ta and W-2Re-1Ta alloys using nanoindentation and APT has been studied by $\mathrm{Xu}$ et al. [81]. The W-2Re alloy showed an increase of around $3.3 \mathrm{GPa}$ and 3.8 $\mathrm{GPa}$ at irradiation temperatures of 300 and $500^{\circ} \mathrm{C}$ respectively, agreeing well with the work of Armstrong et al. [79]. Xu et al. [81] attributed the increase in hardness between the two irradiation temperatures solely to increased Re precipitation at higher temperatures by assuming the hardness 
increase due to dislocation loops saturates at 1.0 GPa from Ref. [79] although no detailed analysis of the damage microstructure is given.

Gibson et al. [80] performed nanoindentation studies on $\mathrm{W}$ samples irradiated with $2 \mathrm{MeV} \mathrm{He}$ ions to concentrations of $\sim 600 \mathrm{appm} \mathrm{He}$ and $\sim 0.02 \mathrm{DPA}$ at an irradiation temperature of $800^{\circ} \mathrm{C}$. The authors noted an increased in the hardness of $0.9 \mathrm{GPa}$ agreeing well with previous works on $\mathrm{He}$ ion irradiated materials at lower temperatures $\left(300^{\circ} \mathrm{C}\right)$ [82]. Nanoindentation has also been used to measure the hardness increase of $200 \mathrm{keV}$ He ion irradiated W samples at temperatures of RT, 200, 400 and $800^{\circ} \mathrm{C}$ to a peak damage of 0.5 DPA and $~ 30,000$ appm He by Cui et al. [83]. The authors [83] attributed the reduced increase in hardness as a function of irradiation temperature to the lower dislocation loop and He-vac complex density as compared by positron annihilation spectroscopy.

Of the hardness data available it appears a combination of W alloys doped with Re, Os and Ta may yield the more promising systems as to model the hardening effects of transmutation products during neutron irradiations [81]. In addition to this, the sequential ion-irradiation experiments using $\mathrm{W}$ followed by He appear to also better match the neutron hardening results [82]. There has also been no combination of the two experiments (W-Re-Os-Ta based alloys and dual ion beam irradiations) which may yet reproduce the neutron results more accurately. Furthermore, the lack of cavities observed in the works with He implantation $[80,82]$ may still be hindering the comparison, as voids are routinely observed in neutron experiments and have been noted to play a significant role in the irradiation hardening at lower doses [41]. However, no simultaneous ion beam irradiation (self-ion $+\mathrm{He} / \mathrm{H}$ ) data exists for $\mathrm{W}$ or $\mathrm{W}-\mathrm{Re}$ alloys which may be a promising avenue to pursue in the replication of hardening mechanisms arising from neutron irradiations. It is also now possible, with the careful consideration of irradiating projectile (as to better match the PKA spectrum in a neutron irradiation), alloy composition and high DPAs to form $\sigma$ and $\chi$ phase precipitation under ion irradiated W26Re alloys [88] which will undoubtedly lead to better emulation of the hardness increases observed in neutron irradiated W.

\subsection{Thermal diffusivity}


Under irradiating condition the thermal diffusivity of materials decreases due to the increase in the number of defects present in the material leading to enhanced phonon and electron scattering events [17]. Figure 15 shows a plot of thermal diffusivity as a function of temperature for $\mathrm{W}$ samples exposed to neutron and ion irradiations [91,92]. At temperatures lower than $1000^{\circ} \mathrm{C}$ the electrical and thermal conductivity of $\mathrm{W}$ have been shown to decrease with increasing irradiation dose due to the increase in these scattering events [93-95]. Roedig et al. [91,96] measured the thermal diffusivities of $\mathrm{W}-1 \% \mathrm{La}_{2} \mathrm{O}_{3}$ blocks after fast neutron irradiation at temperatures of either 200,350 or $700^{\circ} \mathrm{C}$ up to 0.6 DPA in a mixed neutron spectra at the High Flux Reactor (HFR), in Petten, Netherlands. The authors noted a significant decrease in the thermal diffusivity with increasing irradiation dose; however, this became much less significant at higher temperatures $\left(>1000^{\circ} \mathrm{C}\right)$ which is attributed to the annihilation of defects and thus, scattering points which is in good agreement with the microstructures of neutron [30-32] and He ion irradiated [49] W showing low dislocation loop density at temperatures of $>800^{\circ} \mathrm{C}$ and $1000^{\circ} \mathrm{C}$, respectively.

Thermal conduction is a contribution of phonon and electron conduction. However, in metals at temperatures above the Debye temperature the thermal conductivity is dominated by electron conduction. Thus, the work of Roedig [91] is also in good agreement with the recovery of electrical conductivity measurements of neutron irradiated materials where complete defect recovery should occur at temperatures of around $1200^{\circ} \mathrm{C}$ with any residual electrical resistivity (compared to the pristine material) arising from Re precipitates [93]. Fujitsuka et al. [97] have examined the effect of neutron irradiation on the thermal diffusivity of $\mathrm{W}$ and $\mathrm{W}$-Re alloys irradiated in the JMTR at $\sim 50^{\circ} \mathrm{C}$. The authors noted a decrease from around $70 \mathrm{~mm}^{2} / \mathrm{s}$ to $61 \mathrm{~mm}^{2} / \mathrm{s}(\sim 15 \%)$ after irradiation when measured at room temperature but, only a decrease of $\sim 9 \%$ when measured at around $700^{\circ} \mathrm{C}$ agreeing well with the recovery discussed above [17].

Measurements of the change in thermal conductivity of ion-irradiated W (and ion irradiated materials in general) are sparse, mostly due to the challenges in measuring the shallow damage layer due to the nature of ion irradiation and lack of suitable techniques. The 3- $\omega[98]$ and thermoreflectance 
techniques [99] have very recently been used to measure the change in thermal conductivity of $\mathrm{He}$ plasma irradiated $\mathrm{W}$, however, these studies are not considered further in this review due to their sub-threshold displacement energies giving rise to drastically different damage features as compared with neutrons. The change in thermal diffusivity of self-ion irradiated W samples irradiated to 0.25 DPA and sequential implantation with 300 and 3100 appm He (using between 50keV to $2 \mathrm{MeV} \mathrm{He}$ ) at $300^{\circ} \mathrm{C}$ using a non-contact laser-induced transient grating technique has been performed by Hofmann et al. [92]. The authors noted a drastic decrease in the thermal diffusivity of around $30 \%$ for the sample implanted with 300 appm He, when measured at room temperature. Although high temperature data is lacking for this study, from Figure 15 it can be seen that the technique employed by Hofmann et al. [92] matched well with that of the laser flash method used by Roedig et al. [91] for the unirradiated material. It is clear from Figure 15 that the implantation of He can emulate the decrease in the thermal diffusivity of $\mathrm{W}$ samples exposed to neutron irradiation. Hofmann et al. [92] attributed this to the scattering effect of a He containing vacancy which has similar scattering to an empty vacancy, as the He doesn't contribute valence electrons [100]. The dramatic decrease in the higher dose He implantation (3000 appm He) may be due to the large number of scattering points. 


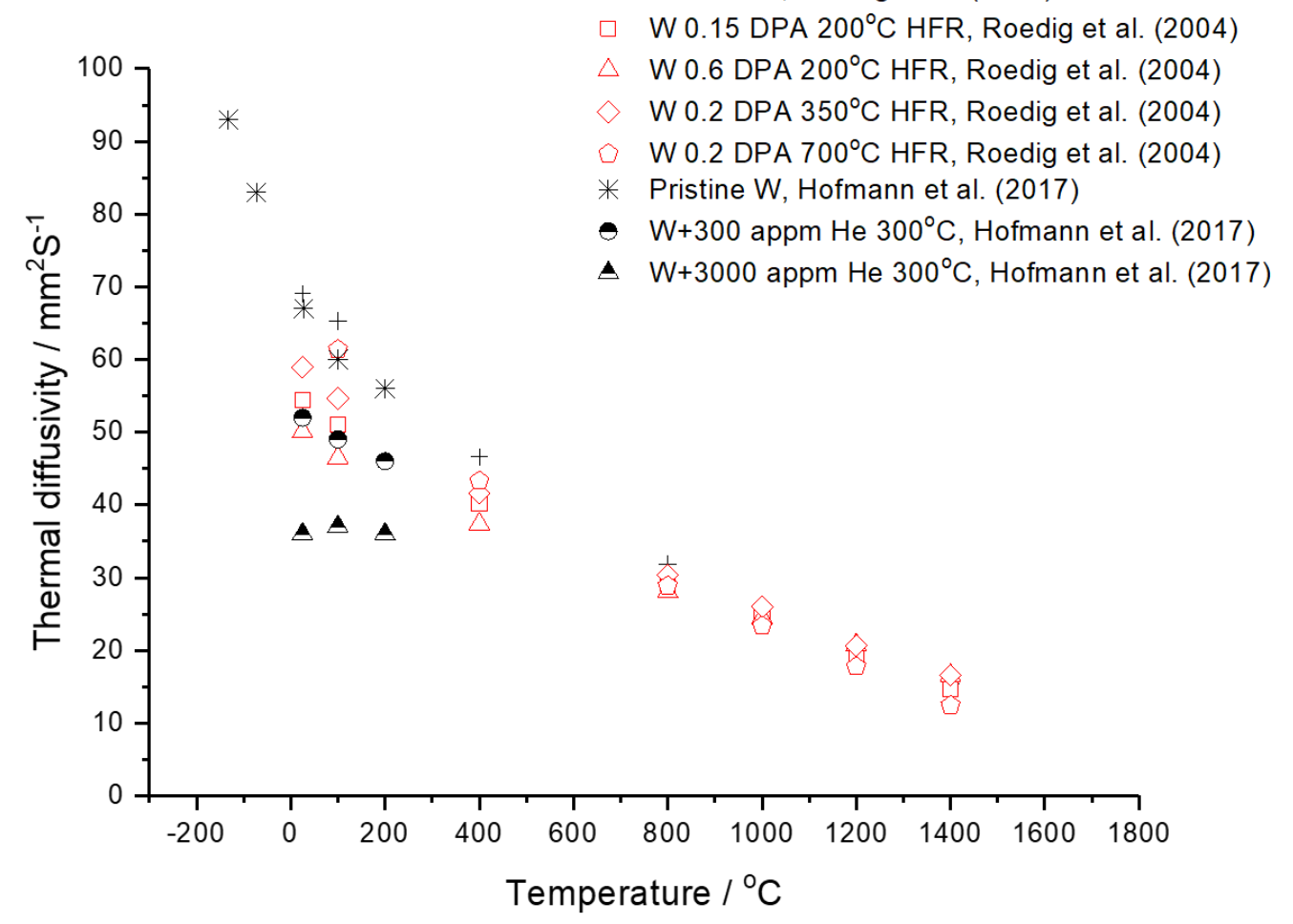

\section{Figure 15. Thermal diffusivity as a function temperature for neutron [91] (open red symbols) and He ion irradiated W samples [92] (semi-closed black symbols).}

\section{Conclusions and outlook}

This review has highlighted key areas of comparison in the effort to emulate neutron irradiation damage with ion beam(s). Many aspects of neutron irradiation damage microstructures and properties can be reproduced from ion beams, such as; dislocation loop types and sizes, cavity lattices and degradation in thermophysical properties. Typically, however, only one aspect of neutron irradiation damage may be reproduced under a given ion irradiation schedule, with the complex synergistic effects of dense cascades, gas impurity atoms and transmutation products during neutron irradiations still seemingly difficult to obtain. It has been found that for the formation of energetically unfavourable $\boldsymbol{b}=\langle 100\rangle$ dislocation loops, high energy self-ion irradiations promote their formation via high energy density cascade formation. Continued work into the effect of cascade energy density on the fraction of $\boldsymbol{b}=\langle 100\rangle$ dislocation loops would be warranted to further understand their 
formation under differing PKA spectra as to predict their formation under reactor types (thermal, fast and fusion neutron spectra).

Furthermore, high energy self-ion irradiations of W alloys with the addition of metallic transmutation products ( $\mathrm{Re}, \mathrm{Os}$ and $\mathrm{Ta}$ ) has provided insight into the effects of intermetallic clustering on the mechanical degradation during irradiation; however, it has not yet been fully and accurately captured by ion beam experiments. This may be due to the much higher possible PKA energies in these selfion irradiations. Recent ion beam irradiations have shown that, with careful consideration of the irradiating projectile and energy (as to better mimic the PKA spectrum of a FBR neutron reactor) has led to the novel observation of non-equilibrium intermetallic $\sigma$ and $\chi$ phase precipitates under ion irradiation. Thus, further work is needed to clarify the effects of projectile mass and energy, Re content and total DPA on the rate of precipitation in WRe alloys. This will allow for a more fundamental understanding of this complex non-equilibrium phase formation under irradiation and determine the ability of ion irradiation to physically model neutron irradiation hardening in $\mathrm{W}$ and W alloys.

Other damage features, such as voids and cavities have yet to be accurately reproduced using heavy/self-ion irradiation alone (i.e. dislocation loop populations). Contrary to this, light ion irradiations using $\mathrm{H}$ and $\mathrm{He}$ ions have proved effective in emulating cavity formation and superlattice structures but differ in their reproduction of dislocation loops type and nature due to light ion irradiations producing low energy density, dilute cascades composed of many isolated FPs. Thus, a combination of all these efforts may be foreseen in the pursuit of a more effective way to reproduce more/all aspects of neutron irradiation damage with ion beam(s); irradiation of $\mathrm{W}$ and $\mathrm{W}$ alloys doped with transmutation products under high energy ion irradiations, designed to better mimic the PKA spectrum and maximum PKA energies from neutron irradiations, combined with simultaneous light-ion ( $\mathrm{H}$ or $\mathrm{He}$ to represent gas transmutation atoms) implantation to capture void and void superlattice formation. In addition to this, reproducing the pioneering and extensive work on 
dislocation loop type and nature in the ion irradiation experiments of $\mathrm{W}$ with neutron irradiated samples would undoubtedly prove invaluable to assist in the validation of ion beam experiments.

\section{Acknowledgments}

The author is very grateful to Drs S. Humphry-Baker and P.D. Edmondson and Prof. S.E. Donnelly for their fruitful discussion. 


\section{References}

[1] H. Bolt, V. Barabash, W. Krauss, J. Linke, R. Neu, S. Suzuki, N. Yoshida, A.U. Team, Materials for the plasma-facing components of fusion reactors, 2004. doi:10.1016/j.jnucmat.2004.04.005.

[2] D.S. Darrow, F.E. Cecil, V. Kiptily, K. Fullard, A. Horton, A. Murari, Observation of alpha particle loss from JET plasmas during ion cyclotron resonance frequency heating using a thin foil Faraday cup detector array, Rev. Sci. Instrum. 81 (2010) $10 \mathrm{D} 330$. doi:10.1063/1.3502325.

[3] M.R. Gilbert, J.-C. Sublet, Neutron-induced transmutation effects in W and W-alloys in a fusion environment, Nucl. Fusion. 51 (2011) 43005. http://stacks.iop.org/0029$5515 / 51 / \mathrm{i}=4 / \mathrm{a}=043005$.

[4] M.R. Gilbert, S. Zheng, R. Kemp, L.W. Packer, S.L. Dudarev, J.-C. Sublet, Comparative Assessment of Material Performance in DEMO Fusion Reactors, Fusion Sci. Technol. 66 (2014) 9-17. doi:10.13182/FST13-751.

[5] G.S. Was, Fundamentals of Radiation Materials Science, 1st ed., Springer-Verlag Berlin Heidelberg, New York, 2007. doi:10.1017/CBO9781107415324.004.

[6] L.K. Mansur, Theory and experimental background on dimensional changes in irradiated alloys, J. Nucl. Mater. 216 (1994) 97-123. doi:https://doi.org/10.1016/00223115(94)90009-4.

[7] L.K. Mansur, Correlation of neutron and heavy-ion damage: II. The predicted temperature shift if swelling with changes in radiation dose rate, J. Nucl. Mater. 78 (1978) 156-160. doi:https://doi.org/10.1016/0022-3115(78)90514-7.

[8] N.H. Packan, K. Farrell, J.O. Stiegler, Correlation of neutron and heavy-ion damage: I. The influence of dose rate and injected helium on swelling in pure nickel, J. Nucl. Mater. 78 (1978) 143-155. doi:https://doi.org/10.1016/0022-3115(78)90513-5.

[9] S.J. Zinkle, L.L. Snead, Opportunities and limitations for ion beams in radiation effects studies: Bridging critical gaps between charged particle and neutron irradiations, Scr. Mater. 143 (2018) 154-160. doi:https://doi.org/10.1016/j.scriptamat.2017.06.041.

[10] W.G. Johnston, J.H. Rosolowski, A.M. Turkalo, T. Lauritzen, The depth distribution of void swelling produced by $5 \mathrm{MeV}$ Ni ions, J. Nucl. Mater. 62 (1976) 167-180. doi:https://doi.org/10.1016/0022-3115(76)90014-3.

[11] B.N. Singh, A. Horsewell, Effects of fission neutron and $600 \mathrm{MeV}$ proton irradiations on microstructural evolution in OFHC-copper, J. Nucl. Mater. 212-215 (1994) 410-415. doi:https://doi.org/10.1016/0022-3115(94)90095-7.

[12] S.J. Zinkle, B.N. Singh, Microstructure of $\mathrm{Cu}-\mathrm{Ni}$ alloys neutron irradiated at $210^{\circ} \mathrm{C}$ and $420^{\circ} \mathrm{C}$ to $14 \mathrm{dpa}$, J. Nucl. Mater. 283-287 (2000) 306-312. doi:https://doi.org/10.1016/S0022-3115(00)00359-7.

[13] P.J. Doyle, K.M. Benensky, S.J. Zinkle, Modeling the impact of radiation-enhanced diffusion on implanted ion profiles, J. Nucl. Mater. 509 (2018) 168-180. doi:10.1016/j.jnucmat.2018.06.042.

[14] J.F. Ziegler, Stopping of energetic light ions in elemental matter, J. Appled Phys. 85 (1999) 1249. doi:http://dx.doi.org/10.1063/1.369844. 
[15] J.A. Hinks, Transmission electron microscopy with in situ ion irradiation, J. Mater. Res. 30 (2015) 1214-1221. doi:10.1557/jmr.2014.384.

[16] J. Fikar, R. Gröger, R. Schäublin, Interaction of irradiation-induced prismatic dislocation loops with free surfaces in tungsten, Nucl. Instruments Methods Phys. Res. Sect. B Beam Interact. with Mater. Atoms. 393 (2017) 186-189. doi:https://doi.org/10.1016/j.nimb.2016.10.006.

[17] G. Pintsuk, Tungsten as a Plasma-Facing Material, in: Compr. Nucl. Mater., Elsevier, 2012: pp. 551-581. doi:10.1016/B978-0-08-056033-5.00118-X.

[18] R.G. Abernethy, Predicting the performance of tungsten in a fusion environment: a literature review, Mater. Sci. Technol. 33 (2017) 388-399. doi:10.1080/02670836.2016.1185260.

[19] H. Xu, R.E. Stoller, Y.N. Osetsky, D. Terentyev, Solving the puzzle of 100 Interstitial Loop Formation in bcc Iron, Phys. Rev. Lett. 110 (2013). doi:10.1103/PhysRevLett.110.265503.

[20] X. Yi, M.L. Jenkins, K. Hattar, P.D. Edmondson, S.G. Roberts, Characterisation of radiation damage in $\mathrm{W}$ and $\mathrm{W}$-based alloys from $2 \mathrm{MeV}$ self-ion near-bulk implantations, Acta Mater. 92 (2015) 163-177. doi:10.1016/j.actamat.2015.04.015.

[21] A. Prokhodtseva, B. Décamps, A. Ramar, R. Schäublin, Impact of He and Cr on defect accumulation in ion-irradiated ultrahigh-purity Fe(Cr) alloys, Acta Mater. 61 (2013) 69586971. doi:10.1016/j.actamat.2013.08.007.

[22] D. Nguyen-Manh, S.L. Dudarev, A.P. Horsfield, Systematic group-specific trends for point defects in bec transition metals: An ab initio study, J. Nucl. Mater. 367-370 A (2007) 257262. doi:10.1016/j.jnucmat.2007.03.006.

[23] B.L. Eyre, R. Bullough, On the formation of interstitial loops in b.c.c. metals, Philos. Mag. A J. Theor. Exp. Appl. Phys. 12 (1965) 31-39. doi:10.1080/14786436508224943.

[24] C.A. English, M.L. Jenkins, Molecular ion irradiations of molybdenum, Philos. Mag. 90 (2010) 821-843. doi:10.1080/14786430903217826.

[25] W. Jäger, M. Wilkens, Formation of vacancy-type dislocation loops in tungsten bombarded by $60 \mathrm{keV}$ Au ions, Phys. Status Solidi. 32 (1975) 89-100. doi:10.1002/pssa.2210320109.

[26] B.C. Masters, Dislocation loops in irradiated iron, Philos. Mag. A J. Theor. Exp. Appl. Phys. 11 (1965) 881-893. doi:10.1080/14786436508223952.

[27] A.E. Sand, S.L. Dudarev, K. Nordlund, High-energy collision cascades in tungsten: Dislocation loops structure and clustering scaling laws, EPL (Europhysics Lett. 103 (2013) 46003. http://stacks.iop.org/0295-5075/103/i=4/a=46003.

[28] N. Castin, G. Bonny, A. Bakaev, C.J. Ortiz, A.E. Sand, D. Terentyev, Object kinetic Monte Carlo model for neutron and ion irradiation in tungsten: Impact of transmutation and carbon impurities, J. Nucl. Mater. 500 (2018) 15-25. doi:https://doi.org/10.1016/j.jnucmat.2017.12.014.

[29] G. Bonny, N. Castin, A. Bakaev, D. Terentyev, Kinetic Monte Carlo model for 1-D migration in a field of strong traps: Application to self-interstitial clusters in W-Re alloys, Comput. Mater. Sci. 144 (2018) 355-362. doi:https://doi.org/10.1016/j.commatsci.2017.12.024.

[30] A. Hasegawa, M. Fukuda, S. Nogami, K. Yabuuchi, Neutron irradiation effects on tungsten 
materials, Fusion Eng. Des. 89 (2014) 1568-1572. doi:10.1016/j.fusengdes.2014.04.035.

[31] A. Hasegawa, M. Fukuda, K. Yabuuchi, S. Nogami, Neutron irradiation effects on the microstructural development of tungsten and tungsten alloys, J. Nucl. Mater. 471 (n.d.) 175-183. doi:10.1016/j.jnucmat.2015.10.047.

[32] M. Fukuda, K. Yabuuchi, S. Nogami, A. Hasegawa, T. Tanaka, Microstructural development of tungsten and tungsten-rhenium alloys due to neutron irradiation in HFIR, J. Nucl. Mater. 455 (2014) 460-463. doi:10.1016/j.jnucmat.2014.08.002.

[33] T. Tanno, A. Hasegawa, J.-C. He, M. Fujiwara, S. Nogami, M. Satou, T. Shishido, K. Abe, Effects of Transmutation Elements on Neutron Irradiation Hardening of Tungsten, Mater. Trans. 48 (2007) 2399-2402. doi:10.2320/matertrans.MAW200722.

[34] T. Tanno, a. Hasegawa, J.C. He, M. Fujiwara, M. Satou, S. Nogami, K. Abe, T. Shishido, Effects of transmutation elements on the microstructural evolution and electrical resistivity of neutron-irradiated tungsten, J. Nucl. Mater. 386-388 (2009) 218-221. doi:10.1016/j.jnucmat.2008.12.091.

[35] V.K. Sikka, J. Moteff, Superlattice of voids in neutron-irradiated tungsten, J. Appl. Phys. 43 (1972) 4942-4944. doi:10.1063/1.1661050.

[36] A. Hasegawa, T. Tanno, S. Nogami, M. Satou, Property change mechanism in tungsten under neutron irradiation in various reactors, J. Nucl. Mater. 417 (2011) 491-494. doi:10.1016/j.jnucmat.2010.12.114.

[37] M. Ekman, K. Persson, G. Grimvall, Phase diagram and lattice instability in tungstenrhenium alloys, J. Nucl. Mater. 278 (2000) 273-276. doi:https://doi.org/10.1016/S00223115(99)00241-X.

[38] A. Hasegawa, M. Fukuda, T. Tanno, S. Nogami, Neutron Irradiation Behavior of Tungsten, Mater. Trans. 54 (2013) 466-471. doi:10.2320/matertrans.MG201208.

[39] I. Ipatova, R.W. Harrison, D. Terentyev, S.E. Donnelly, E. Jimenez-Melero, Thermal Evolution of the Proton Irradiated Structure in Tungsten $-5 \mathrm{wt} \%$ Tantalum, J. Fusion Energy. 36 (2017) 234-239. doi:10.1007/s10894-017-0145-y.

[40] T. Koyanagi, N.A.P.K. Kumar, T. Hwang, L.M. Garrison, X. Hu, L.L. Snead, Y. Katoh, Microstructural evolution of pure tungsten neutron irradiated with a mixed energy spectrum, J. Nucl. Mater. 490 (2017) 66-74. doi:10.1016/j.jnucmat.2017.04.010.

[41] X. Hu, T. Koyanagi, M. Fukuda, N.A.P.K. Kumar, L.L. Snead, B.D. Wirth, Y. Katoh, Irradiation hardening of pure tungsten exposed to neutron irradiation, J. Nucl. Mater. 480 (2016) 235-243. doi:10.1016/j.jnucmat.2016.08.024.

[42] R.C. Rau, Vacancy dislocation loops in irradiated and annealed tungsten, Philos. Mag. A J. Theor. Exp. Appl. Phys. 18 (1968) 1079-1084. doi:10.1080/14786436808227526.

[43] R.C. Rau, F.S. D'aragona, R.L. Ladd, Neutron damage in molybdenum irradiated at high temperatures, Philos. Mag. A J. Theor. Exp. Appl. Phys. 21 (1970) 441-452. doi:10.1080/14786437008238430.

[44] X. Yi, M.L. Jenkins, M. Briceno, S.G. Roberts, Z. Zhou, In situ study of self-ion irradiation damage in $\mathrm{W}$ and $\mathrm{W}-5 \operatorname{Re}$ at $500^{\circ} \mathrm{C}$, Philos. Mag. A. 93 (2013) 1715-1738. doi:10.1080/14786435.2012.754110.

[45] X. Yi, M.L. Jenkins, M.A. Kirk, Z. Zhou, S.G. Roberts, In-situ TEM studies of $150 \mathrm{keV}$ 
$\mathrm{W}+$ ion irradiated $\mathrm{W}$ and $\mathrm{W}$-alloys: Damage production and microstructural evolution, Acta Mater. 112 (2016) 105-120. doi:10.1016/j.actamat.2016.03.051.

[46] F. Ferroni, X. Yi, K. Arakawa, S.P. Fitzgerald, P.D. Edmondson, S.G. Roberts, High temperature annealing of ion irradiated tungsten, Acta Mater. 90 (2015) 380-393. doi:10.1016/j.actamat.2015.01.067.

[47] J.C. He, a. Hasegawa, K. Abe, Effects of transmutation elements on the defect structure development of W irradiated by protons and neutrons, J. Nucl. Mater. 377 (2008) 348-351. doi:10.1016/j.jnucmat.2008.03.014.

[48] I. Ipatova, R.W. Harrison, P.T. Wady, S.M. Shubeita, D. Terentyev, S.E. Donnelly, E. Jimenez-Melero, Structural defect accumulation in tungsten and tungsten-5wt.\% tantalum under incremental proton damage, J. Nucl. Mater. (2017). doi:10.1016/j.jnucmat.2017.11.030.

[49] R.W. Harrison, G. Greaves, J.A. Hinks, S.E. Donnelly, A study of the effect of helium concentration and displacement damage on the microstructure of tungsten irradiation with helium ions, J. Nucl. Mater. 495 (2017) 492-503.

[50] K. Krishan, Invited review article ordering of voids and gas bubbles in radiation environments, Radiat. Eff. 66 (1982) 121-155. doi:10.1080/00337578208222474.

[51] O. El-Atwani, J.A. Hinks, G. Greaves, S. Gonderman, T. Qiu, M. Efe, J.P. Allain, In-situ TEM observation of the response of ultrafine- and nanocrystalline-grained tungsten to extreme irradiation environments., Sci. Rep. 4 (2014) 4716. doi:10.1038/srep04716.

[52] I. Ipatova, P.T. Wady, S.M. Shubeita, C. Barcellini, A. Impagnatiello, E. Jimenez-Melero, Radiation-induced void formation and ordering in Ta-W alloys, J. Nucl. Mater. 495 (2017) 343-350. doi:10.1016/j.jnucmat.2017.08.029.

[53] F. Luo, L. Guo, D. Lu, J. Wang, Z. Zheng, W. Zhang, Z. Shen, TEM observation of bubbles induced by single and sequential He/H irradiation in tungsten, Fusion Eng. Des. 125 (2017) 463-467. doi:10.1016/j.fusengdes.2017.04.014.

[54] R.W. Harrison, G. Greaves, J.A. Hinks, S.E. Donnelly, A study of the effect of helium concentration and displacement damage on the microstructure of helium ion irradiated tungsten, J. Nucl. Mater. 495 (2017). doi:10.1016/j.jnucmat.2017.08.033.

[55] X. Yi, M.L. Jenkins, M. Briceno, S.G. Roberts, Z. Zhou, M.A. Kirk, In-situ study of selfion irradiation damage in $\mathrm{W}$ and $\mathrm{W}-5 \mathrm{Re}$ at $500^{\circ} \mathrm{C}$, Philos. Mag. A. 93 (2012) 1715-1738.

[56] T. Matsui, S. Muto, T. Tanabe, TEM study on deuterium-irradiation-induced defects in tungsten and molybdenum, J. Nucl. Mater. 283-287 (2000) 1139-1143. doi:10.1016/S0022-3115(00)00169-0.

[57] R.W. Harrison, H. Amari, G. Greaves, J.A. Hinks, S.E. Donnelly, Effect of He-appm/DPA ratio on the damage microstructure of tungsten, MRS Adv. 1 (2016) 2893-2899. doi:10.1557/adv.2016.385.

[58] R. Schäublin, B. Décamps, A. Prokhodtseva, J.F. Löffler, On the origin of the primary $1 / 2$ a0<111> and a0<100> loops in irradiated Fe(Cr) alloys, Acta Mater. 133 (2017) 427-439. doi:10.1016/j.actamat.2017.02.041.

[59] R.W. Harrison, J.A. Hinks, S.E. Donnelly, Influence of pre-implanted helium on dislocation loop type in tungsten under self-ion irradiation, Scr. Mater. 150 (2018) 61-65. 
[60] R.W. Harrison, G. Greaves, J.A. Hinks, S.E. Donnelly, Engineering self-organising helium bubble lattices in tungsten, Sci. Rep. 7 (2017) 7724.

[61] P.B. Johnson, D.J. Mazey, J.H. Evans, Bubble structures in He + irradiated metals, Radiat. Eff. 78 (1983) 147-156. doi:10.1080/00337578308207367.

[62] J.H.J. Evans, Simulations of the effects of 2-D interstitial diffusion on void lattice formation during irradiation, Philos. Mag. 86 (2006) 173-188. doi:10.1080/14786430500380134.

[63] P.B. Johnson, Gas Bubble Lattices in Metals - Fundamental Aspects of Inert Gases in Solids, in: S.E. Donnelly, J.H. Evans (Eds.), Springer US, Boston, MA, 1991: pp. 167-184. doi:10.1007/978-1-4899-3680-6_14.

[64] J.H. Evans, Observations of a Regular Void Array in High Purity Molybdenum irradiated with $2 \mathrm{MeV}$ Nitrogen Ions, Nature. 229 (1971) 403-404. http://dx.doi.org/10.1038/229403a0.

[65] P.B. Johnson, D.J. Mazey, Helium gas bubble lattices in face-centred-cubic metals, Nature. 276 (1978) 595-596. http://dx.doi.org/10.1038/276595a0.

[66] P.B.P. Johnson, D.J.D. Mazey, Gas-bubble superlattice formation in bcc metals, J. Nucl. Mater. 218 (1995) 273-288. doi:10.1016/0022-3115(94)00674-1.

[67] V.I. Dubinko, A. V. Tur, A.A. Turkin, V. V. Yanovskij, A mechanism of formation and properties of the void lattice in metals under irradiation, J. Nucl. Mater. 161 (1989) 57-71. doi:10.1016/0022-3115(89)90462-5.

[68] C.H. Woo, W. Frank, A Theory of Void Lattice Formation, J. Nucl. Mater. 137 (1985) 7 21.

[69] J.H.J. Evans, Void and bubble lattice formation in molybdenum: A mechanism based on two-dimensional self-interstitial diffusion, J. Nucl. Mater. 119 (1983) 180-188. doi:10.1016/0022-3115(83)90195-2.

[70] D.J. Mazey, B.L. Eyre, J.H. Evans, S.K. Erents, G.M. McCracken, A transmission electron microscopy study of molybdenum irradiated with helium ions, J. Nucl. Mater. 64 (1977) 145-156. doi:10.1016/0022-3115(77)90018-6.

[71] J.H.J. Evans, A computer simulation of the two-dimensional SIA diffusion model for void lattice formation, J. Nucl. Mater. 132 (1985) 147-155. doi:10.1016/0022-3115(85)90408-8.

[72] J.H. Evans, Irradiation-Induced Cavity Lattice Formation in Metals, in: D. Walgraef, N.M. Ghoniem (Eds.), Patterns, Defects Mater. Instab., Springer Netherlands, Dordrecht, 1990: pp. 347-370. doi:10.1007/978-94-009-0593-1_23.

[73] D. Nguyen-Manh, A.P. Horsfield, S.L. Dudarev, Self-interstitial atom defects in bcc transition metals: Group-specific trends, Phys. Rev. B. 73 (2006) 20101. http://link.aps.org/doi/10.1103/PhysRevB.73.020101.

[74] D.J. Mazey, S. Francis, J.A. Hudson, Observation of a partially-ordered void lattice in aluminium irradiated with $400 \mathrm{keV}$ Al+ ions, J. Nucl. Mater. 47 (1973) 137-142. doi:https://doi.org/10.1016/0022-3115(73)90095-0.

[75] C.-H. Huang, M.R. Gilbert, J. Marian, Simulating irradiation hardening in tungsten under fast neutron irradiation including Re production by transmutation, J. Nucl. Mater. 499 (2018) 204-215. doi:https://doi.org/10.1016/j.jnucmat.2017.11.026. 
[76] J. Marian, C.S. Becquart, C. Domain, S.L. Dudarev, M.R. Gilbert, R.J. Kurtz, D.R. Mason, K. Nordlund, A.E. Sand, L.L. Snead, T. Suzudo, B.D. Wirth, Recent advances in modeling and simulation of the exposure and response of tungsten to fusion energy conditions, Nucl. Fusion. 57 (2017) 92008. http://stacks.iop.org/0029-5515/57/i=9/a=092008.

[77] C.-H. Huang, L. Gharaee, Y. Zhao, P. Erhart, J. Marian, Mechanism of nucleation and incipient growth of Re clusters in irradiated W-Re alloys from kinetic Monte Carlo simulations, Phys. Rev. B. 96 (2017) 94108. doi:10.1103/PhysRevB.96.094108.

[78] L. Gharaee, J. Marian, P. Erhart, The role of interstitial binding in radiation induced segregation in W-Re alloys, J. Appl. Phys. 120 (2016) 1-8. doi:10.1063/1.4956377.

[79] D.E.J. Armstrong, X. Yi, E.A. Marquis, S.G. Roberts, Hardening of self ion implanted tungsten and tungsten 5-wt\% rhenium, J. Nucl. Mater. 432 (2013) 428-436. doi:10.1016/j.jnucmat.2012.07.044.

[80] J.S.K.-L. Gibson, S.G. Roberts, D.E.J. Armstrong, High temperature indentation of heliumimplanted tungsten, Mater. Sci. Eng. A. 625 (2015) 380-384. doi:10.1016/j.msea.2014.12.034.

[81] A. Xu, D.E.J. Armstrong, C. Beck, M.P. Moody, G.D.W. Smith, P.A.J. Bagot, S.G. Roberts, Ion-irradiation induced clustering in W-Re-Ta, W-Re and W-Ta alloys: An atom probe tomography and nanoindentation study, Acta Mater. 124 (2017) 71-78. doi:10.1016/j.actamat.2016.10.050.

[82] D.E.J. Armstrong, P.D. Edmondson, S.G. Roberts, Effects of sequential tungsten and helium ion implantation on nano-indentation hardness of tungsten, Appl. Phys. Lett. 102 (2013) 251901. doi:10.1063/1.4811825.

[83] M.H. Cui, Z.G. Wang, L.L. Pang, T.L. Shen, C.F. Yao, B.S. Li, J.Y. Li, X.Z. Cao, P. Zhang, J.R. Sun, Y.B. Zhu, Y.F. Li, Y.B. Sheng, Temperature dependent defects evolution and hardening of tungsten induced by $200 \mathrm{keV}$ He-ions, Nucl. Inst. Methods Phys. Res. B. 307 (2013) 507-511. doi:10.1016/j.nimb.2012.12.083.

[84] D.L. Krumwiede, T. Yamamoto, T.A. Saleh, S.A. Maloy, G.R. Odette, P. Hosemann, Direct comparison of nanoindentation and tensile test results on reactor-irradiated materials, J. Nucl. Mater. 504 (2018) 135-143. doi:10.1016/j.jnucmat.2018.03.021.

[85] W.D. Nix, H. Gao, Indentation size effects in crystalline materials: A law for strain gradient plasticity, J. Mech. Phys. Solids. 46 (1998) 411-425.

[86] A. Kareer, A. Prasitthipayong, D. Krumwiede, D.M. Collins, P. Hosemann, S.G. Roberts, An analytical method to extract irradiation hardening from nanoindentation hardness-depth curves, J. Nucl. Mater. 498 (2018) 274-281. doi:10.1016/j.jnucmat.2017.10.049.

[87] F. Hofmann, D. Nguyen-Manh, M.R. Gilbert, C.E. Beck, J.K. Eliason, A.A. Maznev, W. Liu, D.E.J. Armstrong, K.A. Nelson, S.L. Dudarev, Lattice swelling and modulus change in a helium-implanted tungsten alloy: X-ray micro-diffraction, surface acoustic wave measurements, and multiscale modelling, Acta Mater. 89 (2015) 352-363. doi:10.1016/j.actamat.2015.01.055.

[88] R.W. Harrison, G. Greaves, J.A. Hinks, S.E. Donnelly, Intermetallic Re phases formed in ion irradiated WRe alloy, J. Nucl. Mater. In Press (2018). doi:https://doi.org/10.1016/j.jnucmat.2018.11.021.

[89] E.R. Reese, M. Bachhav, P. Wells, T. Yamamoto, G. Robert Odette, E.A. Marquis, On $\alpha^{\prime}$ 
precipitate composition in thermally annealed and neutron-irradiated $\mathrm{Fe}-9-18 \mathrm{Cr}$ alloys, J. Nucl. Mater. 500 (2018) 192-198. doi:10.1016/j.jnucmat.2017.12.036.

[90] E.R. Reese, N. Almirall, T. Yamamoto, S. Tumey, G.R. Odette, E.A. Marquis, Dose rate dependence of $\mathrm{Cr}$ precipitation in an ion-irradiated Fe18Cr alloy, Scr. Mater. 146 (2018) 213-217. doi:https://doi.org/10.1016/j.scriptamat.2017.11.040.

[91] M. Roedig, W. Kuehnlein, J. Linke, D. Pitzer, M. Merola, E. Rigal, B. Schedler, E. Visca, Post irradiation testing of samples from the irradiation experiments PARIDE 3 and PARIDE 4, J. Nucl. Mater. 329-333 (2004) 766-770. doi:10.1016/j.jnucmat.2004.04.176.

[92] F. Hofmann, D. Nguyen-Manh, D.R. Mason, M.R. Gilbert, S.L. Dudarev, J.K. Eliason, R.A. Duncan, A.A. Maznev, K.A. Nelson, C.E. Beck, W. Liu, Helium-Ion-Implantation in Tungsten: Progress towards a Coherent Understanding of the Damage Formed and its Effects on Properties, Procedia IUTAM. 21 (2017) 78-85. doi:10.1016/j.piutam.2017.03.040.

[93] L.K. Keys, J.P. Smith, J. Moteff, High-Temperature Recovery of Tungsten after Neutron Irradiation, Phys. Rev. 176 (1968) 851-856. doi:10.1103/PhysRev.176.851.

[94] M.S. Anand, B.M. Pande, R.P. Agarwala, Recovery in neutron irradiated tungsten, Radiat. Eff. 39 (1978) 149-155. doi:10.1080/00337577808234468.

[95] I. V Gorynin, V.A. Ignatov, V. V Rybin, S.A. Fabritsiev, V.A. Kazakov, V.P. Chakin, V.A. Tsykanov, V.R. Barabash, Y.G. Prokofyev, Effects of neutron irradiation on properties of refractory metals, J. Nucl. Mater. 191-194 (1992) 421-425. doi:https://doi.org/10.1016/S0022-3115(09)80079-2.

[96] A.T. Peacock, V. Barabash, W. Dänner, M. Rödig, P. Lorenzetto, P. Marmy, M. Merola, B.N. Singh, S. Tähtinen, J. Van Der Laan, C.H. Wu, Overview of recent European materials R\&D activities related to ITER, J. Nucl. Mater. 329-333 (2004) 173-177. doi:10.1016/j.jnucmat.2004.04.008.

[97] M. Fujitsuka, B. Tsuchiya, I. Mutoh, T. Tanabe, T. Shikama, Effect of neutron irradiation on thermal diffusivity of tungsten-rhenium alloys, J. Nucl. Mater. 283-287 (2000) 11481151. doi:10.1016/S0022-3115(00)00170-7.

[98] S. Cui, M. Simmonds, W. Qin, F. Ren, G.R. Tynan, R.P. Doerner, R. Chen, Thermal conductivity reduction of tungsten plasma facing material due to helium plasma irradiation in PISCES using the improved 3-omega method, J. Nucl. Mater. 486 (2017) 267-273. doi:10.1016/j.jnucmat.2017.01.023.

[99] S. Qu, Y. Li, Z. Wang, Y. Jia, C. Li, B. Xu, W. Chen, S. Bai, Z. Huang, Z. Tang, W. Liu, Thermal conductivity measurement of the He-ion implanted layer of $\mathrm{W}$ using transient thermoreflectance technique, J. Nucl. Mater. 484 (2017) 382-385. doi:10.1016/j.jnucmat.2016.11.029.

[100] D. Nguyen-Manh, S.L. Dudarev, Trapping of He clusters by inert-gas impurities in tungsten: First-principles predictions and experimental validation, Nucl. Instruments Methods Phys. Res. Sect. B Beam Interact. with Mater. Atoms. 352 (2015) 86-91. doi:https://doi.org/10.1016/j.nimb.2014.11.097. 


\section{List of figures}

Figure 1. a) Image of the JET reactor with the divertor section highlighted in the box and b) design of a single cassette used in the divertor structure (reproduced from Eurofusion) ........................... 2 Figure 2. Graph showing transmutation products of $\mathrm{W}$ under a $1.6 \mathrm{GW}$ DEMO fusion reactor (neutron spectra $5 \times 10^{14} \mathrm{n} \mathrm{cm}^{-2} \mathrm{~s}^{-1}$ ) for two full power years, reproduced from Ref. [3] .................. 4 Figure 3. Figure showing the different crystallographic orientations of SIAs adopting the splitinterstitial model in the BCC unit cell.

Figure 4. Summary of micrographs from neutron irradiated $W$ represented a function of the irradiation temperature and DPA reproduced from Refs. [30-35]

Figure 5. Summary of micrographs from self-ion irradiated $W$ and in-situ annealing experiments represented a function of the irradiation temperature and DPA reproduced from Refs. [20,44-46]

Figure 6. Plot of dislocation loop densities for neutron irradiated $W$ and WRe alloys (open red symbols, line to guide eyes for neutron data only) $[32,36,41,47]$ and ion irradiated $W$ samples (semi/closed black symbols) [20,46,48,49].

Figure 7. Plot of dislocation loop densities for neutron irradiated $\mathrm{W}$ and WRe alloys (open red symbols, line to guide eyes for neutron data only) $[32,36,41,47]$ and ion irradiated $\mathrm{W}$ samples (semi/closed black symbols) $[20,46,48]$.

Figure 8. Summary of micrographs from He ion irradiated $W$ represented a function of the irradiation temperature and DPA reproduced from Ref. [49]

Figure 9. Plot of cavity (void/gas bubble) diameters for neutron irradiated W (open red symbols, line to guide eyes for neutron data only) $[32,33,35,36,41,47]$ and ion irradiated W samples (semi/closed black symbols) $[39,46,52-54]$.

Figure 10. Plot of cavity (void/gas bubble) densities for neutron irradiated W (open red symbols, line to guide eyes for neutron data only) $[32,33,35,36,41,47]$ and ion irradiated W samples (semi/closed black symbols) $[39,46,52-54]$

Figure 11. a) Diffusion of $<111>$ dumbbell along $<111>$ direction in the BCC unit cell (1-D SIA model), b) diffusion of $\langle 110\rangle$ dumbbell along the (110) plane (2-D SIA diffusion model), c) forbidden rotation of $\langle 110\rangle$ dumbbell onto (011) plane (figure reproduced from Ref. [60]) .27

Figure 12. a) A void lattice in W irradiated with neutrons to a dose of $\sim 1.5$ DPA $\left(\sim 10^{22} \mathrm{n} . \mathrm{cm}^{2}\right)$ at $550^{\circ} \mathrm{C}$ showing ordering along $\{110\}$ planes observed from Ref. [35] and b) a He bubble lattice in W irradiated with $15 \mathrm{keV}$ He ions to a dose of 3.0 DPA $\left(1.1 \times 10^{17}\right.$ ions. $\left.\mathrm{cm}^{2}\right)$ from Ref. [60]........ 28 Figure 13. Hardness increase as a function of dose for neutron irradiated $W$ samples under different conditions $[33,36,41,47]$ (open red symbols) and for ion irradiated $W$ and $W$ alloy samples under different conditions [47,79-83] (closed/semi-filled black symbols). Lines to guide eyes only. ...... 30 Figure 14. (Top row, left to right) BF-TEM image of pure $\mathrm{W}$ irradiated in HFIR showing Re precipitate and dark-field (DF)-TEM images of $\sigma$-phase and $\chi$ phase precipitates [32]. (Bottom row, left to right) BF-TEM image of W26Re alloy irradiated to with $350 \mathrm{keV} \mathrm{Ne}$ ions and DF-TEM images of $\sigma$ and $\chi$ phase precipitates [Ref] .36 Figure 15. Thermal diffusivity as a function temperature for neutron [90] (open red symbols) and He ion irradiated W samples [91] (semi-closed black symbols). .40

\section{$\underline{\text { List of tables }}$}


